\title{
Characterization and pro-inflammatory responses of spore and hyphae samples from various mold species
}

\section{Running title: Mold characterization and inflammatory responses}

Øya $\mathrm{E}^{1}$, Afanou $\mathrm{AKJ}^{2}$, Malla N ${ }^{4}$, Uhlig $\mathrm{S}^{3}$, Rolen $\mathrm{E}^{3}$, Skaar $\mathrm{I}^{3}$, Straumfors $\mathrm{A}^{2}$, Winberg JO $\mathrm{J}^{5}$, Bang $\mathrm{BE}^{4,5}$, Schwarze $\mathrm{PE}^{1}$, Eduard $\mathrm{W}^{2}$, and Holme $\mathrm{JA}^{1 \S}$

${ }^{1}$ Department of Air and Noise, Norwegian Institute of Public Health, Oslo, Norway. ${ }^{2}$ Department for the Chemical and Biological Work Environment, National Institute of Occupational Health, Oslo, Norway. ${ }^{3}$ Norwegian Veterinary Institute, Oslo, Norway. ${ }^{4}$ Department of Occupational and Environmental Medicine, University Hospital of North Norway, Troms $\varnothing$, Norway. ${ }^{5}$ Department of Medical Biology, Faculty of Health Sciences, The Arctic University of Norway, Troms $\emptyset$, Norway.

${ }^{\S}$ Corresponding author: Jørn A. Holme, Department of Air and Noise, Domain of Infection Control and Environment and Health, Norwegian Institute of Public Health, P.O.Box 4404 Nydalen, N-0403 Oslo, Norway. E-mail: jorn.holme@fhi.no; Phone: +47 21076247

\footnotetext{
Abstract

Mold particles from Aspergillus fumigatus, Penicillium chrysogenum, Aspergillus versicolor and Stachybotrys chartarum have been linked to respiratory-related diseases. Here we characterized X-ray-inactivated spores and hyphae fragments from these species by number of particles, morphology, and mycotoxin, $\beta$-glucan and protease content/activity. The proinflammatory properties of mold particles were examined in human bronchial epithelial cells (BEAS-2B) and THP-1 monocytes and phorbol 12-myristate 13-acetate (PMA)-differentiated
} 
THP-1. Spores from P. chrysogenum and S. chartarum contained some hyphae fragments, whereas the other preparations contained either spores or hyphae. Each mold species produced mainly one gelatin degrading protease that was either of the metallo- or serine type, while one remains unclassified. Mycotoxin levels were generally low. Detectable levels of $\beta$-glucans were found mainly in hyphae particle preparations. PMA-differentiated THP-1 macrophages were by far the most sensitive model with effects in the order of $10 \mathrm{ng} / \mathrm{cm}^{2}$. Hyphae preparations of A. fumigatus and P. chrysogenum were more potent than respective spore preparations, whereas the opposite seems to be true for A. versicolor and S. chartarum. Hyphae fragments of $A$. fumigatus, $P$. chrysogenum and A. versicolor enhanced the release of metalloprotease (proMMP-9) most markedly. In conclusion, species, growth stage and characteristics are all important factors for pro-inflammatory potential.

Key words: Mold/Mould particles, cytokines, proteases, mycotoxins, $\beta$-glucans, morphology.

\section{Practical implications:}

Pro-inflammatory effects of mold particles were detected at very low doses/concentrations supporting the notion that mold exposure could be linked to health effects. There were large differences in the pro-inflammatory potential between spores and hyphae fragments, and between various species. There were, however, no direct links between the observed proinflammatory response and sample characteristics including number, size and shape of particles, mycotoxins, $\beta$-glucan and protease content/activity. The results from the present study suggest that more direct analyzes of pro-inflammatory responses of samples from indoor environments should be performed as this may give valuable information to epidemiological studies exploring adverse health effects of indoor dampness/mold. 


\section{Background}

There is sufficient evidence from epidemiological studies of associations between indoor dampness/mold and adverse health effects including respiratory symptoms, respiratory infections and exacerbation of asthma ${ }^{[1,2]}$. Several kinds of indoor air pollution agents may contribute. Mold has been suggested to be particularly important, since it may not only cause infection and toxic effects, but also trigger allergic and non-allergic inflammatory reactions that may be linked to various respiratory-related diseases ${ }^{[3-5]}$. Quantitative guidelines (thresholds) for acceptable levels of indoor contamination of microorganisms/mold have not been suggested ${ }^{[1]}$. However, for the work environment where exposure levels can be much higher, a proposal has been made ${ }^{[6]}$.

Species that commonly occur in moist indoor environments include Aspergillus fumigatus, Penicillium chrysogenum, Aspergillus versicolor and Stachybotrys chartarum. The reproductive spores may disperse into the air. When they deposit they may germinate into multicellular filamentous structures called hyphae, which can grow further into tangled mass of networks known as mycelia. Spores from many species, but not all are easily aerosolized. Spores from some species e.g. S. chartarum are produced in slimy aggregates which are dispersed by water and may become airborne after secondary dispersion ${ }^{[6]}$. Furthermore, experimental studies have demonstrated that not only spores but also hyphae fragments can be liberated from fungal cultures ${ }^{[7-9]}$. Recently, an immune-microscopic method for their detection has been described ${ }^{[10]}$.

In addition to direct microscopic quantification [9], components like ergosterol [11], polysaccharides such as $\beta(1 \rightarrow 3)$-glucans ${ }^{[12]}$ and enzymes such as proteases have been used as measurement units for total fungal exposure. Under certain growth conditions the fungi may 
synthesize and excrete mycotoxins which are active secondary metabolites that are reported to induce toxic and inflammatory effects in experimental studies ${ }^{[13,14]}$. The cell wall of both spores and hyphae consists of a matrix containing e.g. $\beta$-glucans, glycoproteins, and lipids, reinforced by chitin fibrils, but the 3-dimensional structure varies between species and between spores and hyphae ${ }^{[6]}$. Spores are considered to be largely inert towards recognition by the immune system, partly due to the rodlet layer composed of the hydrophobic RodA protein on the surface of resting spores. However, swelling and germination of spores leads to a loss of this protective hydrophobin layer, resulting in an increased exposure of immunological surface components ${ }^{[15,16]}$. Beta-1,3-glucans are constituents of the fungal cell wall, and contain molecular structures called pathogen-associated patterns (PAMPs). PAMPs are recognized by pattern recognition receptors (PRRs) like Toll-like receptors (TLRs), and may thereby induce and/or modify inflammatory responses to antigens/allergens ${ }^{[17]}$.

Furthermore, molds may secrete various proteases that have been linked to different health effects such as chronic inflammation and tissue damage ${ }^{[18,19]}$. Most of the mold proteases belong to either the cysteine $(\mathrm{C})$, serine $(\mathrm{S})$ or metallo $(\mathrm{M})$ class of proteases ${ }^{[20]}$. Matrix metalloproteinases (MMPs) are produced by many cell types, in particular by activated macrophages ${ }^{[21,22]}$. They have broad substrate specificity and can process almost all extracellular matrix proteins as well as non-matrix proteins including cytokines, chemokines, growth factors and cell receptors.

Epithelial cells and alveolar macrophages constitute the first line of defense against inhaled molds ${ }^{[23,24]}$. In addition, monocytes may migrate to the infected site in the alveoli during inflammation ${ }^{[25]}$. The molds are recognized by cellular membrane receptors, including toll-like receptors (TLR), protease-activated receptors (PAR) or C-type lectin receptors (CLR) ${ }^{[26]}$. 
In general, TLR2 and TLR4 are considered to be key recognition components for host innate defense system against fungi ${ }^{[27]}$. TLR5 has been reported to be involved in the immunological response to A. fumigatus in THP-1 monocytes ${ }^{[28]}$, and TLR9 in response to S. chartarum ${ }^{[29]}$. Protease activated receptors (PAR)-2 is a G-protein coupled receptor that can be activated by proteases produced by molds ${ }^{[30]}$. Furthermore, various recognition receptors are found on different cell types and they differentially recognize molds in various growth stages ${ }^{[31-35]}$.

As a response to the activation of different receptors, cells secrete signaling molecules, such as cytokines/chemokines which play a central role in orchestrating the immune response. Proinflammatory cytokines (interleukin (IL)-1 $\beta$, IL-6, IL-8 and tumor necrosis factor; TNF- $\alpha$ ) act on surrounding cells, regulate adhesion molecules, other cytokines and recruit immune cells to the site of infection ${ }^{[36]}$.

A number of studies have characterized biological effects of spores or germinating spores of $A$. fumigatus [31,33,37-39]. However, to our knowledge no other studies have characterized aerosolized spores and hyphae fragments from different fungal species, and tried to link this subsequently to pro-inflammatory responses in human cells.

In the present study spores collected by air- (aerosolized-) and liquid flow (washed spores) and hyphae fragments from A. fumigatus, $P$. chrysogenum, A. versicolor, and S. chartarum were characterized with regard to number, size and shape of particles, occurrence of mycotoxins, $\beta$ 1,3-glucans and proteases. Finally, their pro-inflammatory potentials were examined using human bronchial epithelial cells (BEAS-2B) and THP-1 monocytes and PMA-differentiated THP-1 macrophages as representative lung cell models. 


\section{Materials and Methods}

\section{Fungal samples:}

Three types of fungal samples from four pure fungal isolates were prepared, characterized and used in the present study. Isolates comprised Aspergillus fumigatus Fresenius 1863 (strain A1258 FGSC) purchased from the Fungal Genetics Stock Center (University of Missouri, Kansas City, KS), Aspergillus versicolor Tirobaschi 1908 (strain VI 03554), Penicillium chrysogenum Thom 1910 (strain VI 04528) and Stachybotrys chartarum (Ehrenb) S. Hughes (VI 03618) obtained from the Section of Mycology at the Norwegian Veterinary Institute (Oslo, Norway). The characteristics of these fungal isolates and the procedure for inoculum preparation, except for Stachybotrys chartarum, have been previously described elsewhere ${ }^{[8]}$. Three types of fungal particles (washed and aerosolized spores, and hyphae fragments) were prepared and characterized. Detailed linked to chemicals used and preparation procedures of these particles are presented in Supplementary 1. Cultures age and the media used are summarized in Supplementary 1, Table S1. Several preparations were tested, and similar results obtained (Øya et al. unpublished). Micrographs showing different types of fungal particles in washed spore samples from the four species are presented in Supplementary 1, Figure S1.

\section{Characterization of fungal samples by gravimetry and microscopy}

Hundred microliters of 10-100 fold diluted stock suspensions of the fungal samples were filtrated onto pre-weighed $25 \mathrm{~mm}$ polycarbonate filters $(0.4 \mu \mathrm{m}$ pores $)$ which were dried in a desiccator overnight. The dried filters were weighed once more and the mass of fungal sample determined as the difference between the pre-weight and the final weight. Fungal samples were characterized by mass content ( $\mu \mathrm{g}$ dry weight $/ \mathrm{mL}$ ) and by number of particles (n particles $/ \mathrm{mL}$ ). Spores and fragments present in each sample type were visualized by field emission scanning 
electron microscopy (FESEM SU 6600, Hitachi Ibaraki-Ken, Japan) following filtration of 0.5 $\mathrm{mL}$ of particle suspension (concentration $=100 \mu \mathrm{g} / \mathrm{mL}$ ) through a $37 \mathrm{~mm}$ diameter polycarbonate filter with $0.2 \mu \mathrm{m}$ pore size (Millipore, Tullagreen Cork, Ireland) as previously described ${ }^{[8]}$. Spores and hyphae were recognized by their morphological features and classified in 5 groups based on the number of spores per aggregate and the microscopic length, respectively. At least 200 particles were counted at 3000x magnification and the particle composition was determined by calculating the percentages of each type of particles. Further details are given in Afanou and coworkers ${ }^{[8]}$. The purity of the spore preparation was based on fragment/spore number after microscopic examination; however, estimates based on volume (closer to mass) were also done.

\section{Inactivation of fungal samples}

In order to specifically examine immunologically responses from spores and hyphae, we inactivated the samples. More specifically, fungal samples were irradiated on ice with x-rays $(17,45 \mathrm{~Gy} / \mathrm{min}, 225 \mathrm{kV}, 13 \mathrm{~mA}$, no filter, $5 \mathrm{~cm}$ distance to the source) from X-RAD 225 (Precision X-ray Inc., North Branford) at the Norwegian Institute of Public Health (NIPH) receiving a total dose of $5 \mathrm{kGy}$.

\section{Sample preparation for mycotoxin analyses}

Samples were extracted and analyzed for their respective mycotoxins with Liquid chromatography - high resolution mass spectrometry (LC-HRMS) according to procedures described in Supplementary 2.

Extraction and analysis of $\beta$-glucans in fungal suspensions 
Spores or hyphae fragments (approximately $10^{7}$ spores) were centrifuged and resuspended in $0.05 \%$ Tween 20 and $\beta-1,3$-glucans were extracted by adding equal volume of $0.6 \mathrm{M} \mathrm{NaOH}$ and shaking for $1 \mathrm{~h}$ at room temperature. The extracts were neutralized with equal volume of 0.6 M Tris $\mathrm{HCl}(\mathrm{pH} 7.4)$, and frozen at $-20^{\circ} \mathrm{C}$ until analysis. Beta-1,3-glucans were quantified in duplicates using kinetic rate assay of the Glucatell $\beta$-1,3-glucan detection kit (ACC) according to the manufacturer's descriptions. Different dilutions of the extracts were tested, and possible interfering effects of the sample matrix were controlled by spike recovery tests of each sample using glucan standard.

\section{Characterization of proteases by gelatin and collagen zymography}

Sodium dodecyl sulphate-polyacrylamide gel electrophoresis (SDS-PAGE) was carried out as described previously ${ }^{[40]}$ using $4 \%$ and $10 \%(\mathrm{w} / \mathrm{v})$ polyacrylamide in stacking and separating gel, respectively and $0.1 \%(\mathrm{w} / \mathrm{v})$ of gelatin or collagen in the separating gel. The gel was run at $18 \mathrm{~mA} / \mathrm{gel}$ at $4^{\circ} \mathrm{C}$, washed twice in $2.5 \%$ (v/v) Triton $\mathrm{X}-100$ and stained with $0.2 \%$ Coomassie brilliant blue R-250 (30\% methanol). After destaining (30\% methanol and 10\% acetic acid), proteinase activity was evident as cleared (unstained) regions against the stained background. After electrophoresis, gels were divided into two or more parts, each part washed and incubated in buffer containing either no additives (control), $10 \mathrm{mM}$ EDTA (metalloprotease inhibitor), $1.0 \mathrm{mM}$ Pefabloc (serine protease inhibitor) or $2.8 \mu \mathrm{M}$ E64 (cysteine protease inhibitor). The approximate molecular size of the proteases was determined by comparing their migration distance with the migration distances of proteases with known molecular size. These molecular size markers were; proMMP-9 dimer (225 kDa), proMMP-9 monomer (92 kDa), processed form of MMP-9 (83 kDa); proMMP-2 (72 kDa), processed form of MMP-2 (62 kDa) and $\operatorname{trypsin}(24 \mathrm{kDa})$. 


\section{Cell culture and exposure conditions}

The BEAS-2B cell line, which was established after SV-40 hybrid (Ad12SV40) transformation of human bronchial epithelial cells, was purchased from European Collection of Cell Cultures (ECACC, Salisbury, UK). Cells were maintained in serum-free LHC-9 medium on collagencoated flasks in a humidified atmosphere at $37^{\circ} \mathrm{C}$ with $5 \% \mathrm{CO}_{2}$, with refreshment of medium every second day and passaged twice per week. Two days prior to exposure, cells were plated into collagen-coated $10 \mathrm{~mm} \mathrm{12-well} \mathrm{culture} \mathrm{dishes}\left(8 \times 10^{4}\right.$ cells/well $)$ and grown to near confluence. In all experiments, medium was changed the day after seeding and before exposure. Cells were treated with $0-100 \mu \mathrm{g} / \mathrm{mL}$ fungal preparations (suspended in LHC-9 media) for 24 h. Medium blanks were included as control.

The THP-1 human monocytic leukemia cell line was purchased from American Tissue Type Culture Collection (Rockville, MD, USA), and maintained in a humidified atmosphere at $37^{\circ} \mathrm{C}$ and 5\% $\mathrm{CO}_{2}$, in RPMI 1640 medium, supplemented with $10 \%$ heat-inactivated fetal bovine serum (FBS), $10 \mathrm{mM}$ HEPES, $1 \mathrm{mM}$ pyruvic acid and $0.1 \%$ gentamycin, with refreshment of medium every second day and passaged once per week. On the same day as exposure, cells were plated into $35 \mathrm{~mm} 6$-well culture dishes $\left(8 \times 10^{5}\right.$ cells/well) containing RPMI 1640 medium. In experiments with monocytes cells were treated with $0-100 \mu \mathrm{g} / \mathrm{mL}$ fungal preparations for 24 h, $3 \mathrm{~h}$ after seeding. THP-1 cells $\left(2 \times 10^{5}\right.$ cells/well) were also differentiated into macrophages in $10 \mathrm{~mm}$ 12-well culture dishes containing $1 \mathrm{~mL}$ of RPMI 1640 medium using $200 \mathrm{nM}$ PMA for 3 days. Differentiation of PMA treated cells was enhanced after the initial 3 days stimulus by removing the PMA-containing media then incubating the cells in fresh RPMI 1640 with FBS for additional $3 \mathrm{~h}$. In experiments with macrophages, medium was changed directly before exposure and cells were treated with $0-50 \mu \mathrm{g} / \mathrm{mL}$ fungal particles for $24 \mathrm{~h}$. 
THP-1 monocytes used in MMP analysis were cultured as follows. At the same day as exposure, cells were plated into $10 \mathrm{~mm} 12$-well culture dishes $\left(1.4 \times 10^{6}\right.$ cells/well) containing RPMI 1640 media without FBS. In experiments with monocytes, cells were treated $3 \mathrm{~h}$ after seeding with 0 or $10 \mu \mathrm{g} / \mathrm{mL}$ fungal preparations for $72 \mathrm{~h}$. THP-1 cells $\left(2 \times 10^{5}\right.$ cells/well $)$ were differentiated into macrophages as previously described. In experiments with macrophages, medium was changed to PMA-containing media without FBS immediately before exposure and cells were treated with 0 or $1 \mu \mathrm{g} / \mathrm{mL}$ fungal preparations for $72 \mathrm{~h}$.

\section{Cytotoxicity}

After exposure to the test compounds for $24 \mathrm{~h}$ or $72 \mathrm{~h}$, the cell morphology of the cultures was visually examined with a light microscope. Toxicity was assessed and categorized as the relative amount of floating cells (dead cells) in the cultures versus attached cells (living cells). Cell shape and vacuoles were recognized and documented. Cells were trypsinised and stained with Hoechst 33342 and propidium iodide (PI) for determination of plasma membrane damage, changes in nuclear morphology associated with apoptosis and necrosis, using fluorescence microscopy (Nikon Eclipse E 400 with a 330- to $380 \mathrm{~nm}$ UV-2A excitation filter). A minimum of 300 cells per slide were counted (original magnification $\times 400$ ).

\section{Cytokine release}

After exposure, the medium was harvested and centrifuged at $290 \times \mathrm{g}$ for $10 \mathrm{~min}$ to remove mold and floating cells. The final supernatants were stored at $-80^{\circ} \mathrm{C}$. TNF- $\alpha$ IL- $1 \beta$, IL- 6 and IL- 8 protein levels were determined by sandwich ELISA (Enzyme-Linked ImmunoSorbent Assay) according to the manufacturers' guidelines. Absorbance was quantified using a plate reader (TECAN Sunrise, Phoenix Research Products, Hayward, CA, USA) complete with software (Magellan V 1.10). 


\section{Statistical analysis}

The statistical analysis was performed in GraphPad Prism 7 (GraphPad Software, CA, USA). One-way ANOVA was used to analyze the data sets, and Dunnett's post hoc tests were used to compare groups for one-way ANOVA. In all figures, spores and hyphae were plotted separately in two figures. However, the statistics were done as separate one-way ANOVA for each spore or hyphae (i.e. including all applied concentrations) due to differences in number of experiments performed per species. Therefore, no comparison between the responses to the different species was performed. As indicated in the figure legends, all data were log transformed before performing ANOVA to achieve a normal distribution and to fulfil the assumption of equal standard deviations of all sets of replicates, as recommended by the program developers.

\section{Results}

\section{Composition and particle size distribution of exposure materials}

The exposure materials were characterized with regard to morphology, size and composition by scanning electron microscopy to evaluate the distribution of particle types in all samples. As presented in Table 1, aerosolized spore samples of $P$. chrysogenum had $30 \%$ contamination of hyphae fragments, whereas the spore preparation from $S$. chartarum contained a mixture of spores and hyphae fragments with only $26 \%$ spores. However, microscopic examination revealed large spores when compared to small hyphae fragments (Supplementary 1, Figure S1). Therefore, volumes of the different components were estimated by microscopic examination. This estimate which is closer to mass of a sample, gave a contamination of only 8.4 and $21 \%$ hyphae fragments for the spore preparations of P. chrysogenum and S. chartarum, respectively. All other samples were mainly spore (90-97\%) or hyphae fragment (99-100\%) preparations. In 
the spore preparations $87-91 \%$ of the spore particles were single spores, while in the hyphae samples fragments of $1-10 \mu \mathrm{m}(64-84 \%)$ dominated. Washed spores appeared to be smaller and/or have less dry weight than aerosolized spores, thus for the same mass, samples with washed spores contained 2 - 3 times more particles than aerosolized spore preparations.

\section{Characterization of mycotoxin content}

We tested for a large number of relevant mycotoxins by various LC-HRMS analysis. The amounts were in general small and insignificant (Supplementary 3, Figure S2, 3 and 4). In short, we did not find any detectable concentrations of gliotoxin, fumitremorgins A-C, citrin or PRtoxin, penicillin $\mathrm{G}$ or any non-targeted tricothecenes. However, A. versicolor and $P$. chrysogenum contained minor amounts sterigmatocystin and roquefortine $\mathrm{C}$, respectively (Table 2). The mycotoxins detected, seemed to be at somewhat higher levels in washed spores of both species when compared to hyphae fragments.

\section{Measurement of $\beta$-1,3-glucans in fungal samples}

The amounts of $\beta-1,3$-glucans in hyphae fragments were similar in $P$. chrysogenum, $A$. versicolor and S. chartarum, whereas A. fumigatus had somewhat lower amounts (Supplementary 4, Table S2). Washed spores of A. versicolor and S. chartarum also contained some $\beta$-glucans, but much less than hyphae did. Aerosolized spores of $A$. fumigatus and $P$. chrysogenum contained both minor amounts of $\beta$-1,3-glucans.

\section{Characterization of proteases}

Gelatin zymography showed that hyphae fragments of A. fumigatus, P. chrysogenum, and washed spores of $S$. chartarum contained a gelatin degrading protease with molecular sizes of approximately $44 \mathrm{kDa}, 52 \mathrm{kDa}$ and $60 \mathrm{kDa}$, respectively (Figure 1A). Hyphae fragments of $S$. 
chartarum contained one or several proteases with larger molecular size, one just entered the separating gel. The latter had an estimated molecular size of around $300 \mathrm{kDa}$ based on previous experience with proMMP-9/CSPG complexes in THP-1 cells ${ }^{[40,41]}$. However, no gelatin degrading proteases were detected in washed spores of A. fumigatus, P. chrysogenum, A. versicolor and hyphae of $A$. versicolor. Furthermore, none of the species/cellular states contained collagen degrading enzymes (data not shown).

The tested fungal materials contained gelatin degrading proteases that belong to different protease classes. The $44 \mathrm{kDa}$ protease from hyphae fragments of A. fumigatus was partly inhibited by Pefabloc (serine protease inhibitor) but not by EDTA (metalloprotease inhibitor) or E64 (cysteine protease inhibitor) (Figure 1B and C). Thus, this protease is likely a serine protease. The $52 \mathrm{kDa}$ protease from hyphae fragments of $P$. chrysogenum was inhibited by EDTA but not by Pefabloc or E64. Therefore, this protease is deemed a metalloprotease. The $60 \mathrm{kDa}$ protease from washed spores of $S$. chartarum was not stable during the storing conditions and due to the low activity seen in the inhibitory experiments, it was not possible to classify this protease. The $300 \mathrm{kDa}$ protease from hyphae fragments of $S$. chartarum was not inhibited by any of the three inhibitors used and may therefore belong to one of the new families of proteases found in microorganisms or an unassigned family.

\section{Cell viability / Cytotoxicity}

As judged by light microscopy, neither of the test materials seemed to affect viability after neither $24 \mathrm{~h}$ nor $72 \mathrm{~h}$ at these concentrations (not quantified). In addition, neither apoptosis nor necrosis was observed in any of the cell models (data not shown).

Cytokine release 
IL-1 $\beta$ and TNF- $\alpha$ release from BEAS-2B cells was not increased even after $24 \mathrm{~h}$ exposure to $100 \mu \mathrm{g} / \mathrm{mL}$ dry weight mold samples (data not shown). However, at this high concentration marked increases in IL-6 and IL-8 were seen (Supplementary 4, Figure S5). The IL-6 response in $\mathrm{pg} / \mathrm{mL}$ was greater than that of IL-8. The relative potential of the hyphae fragments was $A$. fumigatus $>P$. chrysogenum $>A$. versicolor $>$ S. chartarum. Whereas, the hyphae fragments of $A$. fumigatus and $P$. chrysogenum in general were more potent than the respective spore fraction, responses of $A$. versicolor and $S$. chartarum spores were rather similar to that of their respective hyphae sample.

A. fumigatus and $P$. chrysogenum hyphae fragments induced a marked release of both IL-1 $\beta$ and TNF- $\alpha$, starting at concentrations of 0.1 and $50 \mu \mathrm{g} / \mathrm{mL}$ dry weight in THP-1 monocytes, respectively (Figure 2A). In contrast, none of the other hyphae preparations had any significant effects. Washed spores of A. fumigatus and also A. versicolor induced low, but significant increase in both IL-1 $\beta$ and TNF- $\alpha$ release at concentrations $\leq 50 \mu \mathrm{g} / \mathrm{mL}$ dry weight.

Due to their greater sensitivity, THP-1 macrophages were exposed to mold samples at lower concentrations than the monocytes. All mold preparations significantly induced IL- $1 \beta$ release except aerosolized spores of $P$. chrysogenum (Figure 2B). The relative potential of the hyphae fragments was A. fumigatus $>P$. chrysogenum $>A$. versicolor $>S$. chartarum. A significant induction of both IL-1 $\beta$ and TNF- $\alpha$ by $A$. fumigatus hyphae fragments was observed at concentrations as low as $10 \mathrm{ng} / \mathrm{mL}$. A. fumigatus and A. versicolor washed spores were the most potent of all spores with regard to both IL-1 $\beta$ and TNF- $\alpha$. They induced a significant response at $1 \mu \mathrm{g}$ dry weight $/ \mathrm{mL}$. In THP-1 macrophages, the aerosolized spores of A. fumigatus also significantly increased IL-1 $\beta$. Most interestingly, A. versicolor and S. chartarum spores were at least as potent as their respective hyphae preparations. 


\section{Protease release from THP-1 monocytes and macrophages}

THP-1 monocytes and PMA-differentiated macrophages were treated with the mold samples in order to examine their effect on cellular release of proteases. Based on previously published studies ${ }^{[40,42,43]}$ an exposure period of $72 \mathrm{~h}$ was chosen in these experiments. As can be seen from the data presented in Figure 3A, gelatin zymography of the THP-1 monocyte in serum free conditioned medium showed only a weak band at $92 \mathrm{kDa}$, whereas the medium from cells exposed to washed spores of $A$. fumigatus and $P$. chrysogenum, hyphae fragments of $A$. fumigatus, $P$. chrysogenum, A. versicolor and $S$. chartarum as well as aerosolized spores of $A$. fumigatus and $P$. chrysogenum had increased levels of this gelatin degrading enzyme. The strongest stimulation was observed with hyphae fragments of $A$. fumigatus and $P$. chrysogenum in addition to washed spores of $A$. fumigatus. The $92 \mathrm{kDa}$ band disappeared when the gels were washed and developed in the presence of EDTA (data not shown), and hence this metalloprotease is most likely proMMP-9 as it was previously shown to be the only gelatin degrading enzyme secreted from THP-1 monocytes ${ }^{[42]}$.

Compared to media from THP-1 monocytes, the media from PMA-differentiated THP-1 macrophages showed an increased level of a 92 and a $225 \mathrm{kDa}$ protease (Figure 3B upper panel). As shown previously, these two proteases are the monomer and homodimer forms of proMMP$9^{[41]}$. A very faint band also appeared at $72 \mathrm{kDa}$, the synthesis of which is not affected by incubating the cells with the various mold species. This $72 \mathrm{kDa}$ protease is likely proMMP-2 since these cells have been shown to be able to produce this protease ${ }^{[44]}$. Due to the large amount produced already in the controls of the $92 \mathrm{kDa}$ protease, it was not possible to evaluate if the mold affected the secretion of this protease without dilution of the cell conditioned media. As seen in figure 3B lower panel, in spite of the large induction of the $92 \mathrm{kDa}$ protease following 
PMA-differentiation of THP-1, hyphae fragments from the A. fumigatus, $P$. chrysogenum and A. versicolor could further stimulate the production of this gelatin degrading enzyme. In addition, A. fumigatus washed spores also induced this protease but to a lesser extent. As expected, this band disappeared when the gels were washed and developed in the presence of EDTA (data not shown), confirming that the protease belonged to the metalloprotease family and it is most likely proMMP-9 that has been further induced by the mold.

No collagen degrading enzymes could be detected by collagen zymography in the conditioned media from THP-1 monocytes, macrophages or mold stimulated THP-1 monocytes and macrophages (data not shown).

\section{Discussion}

Spores, crude aqueous extracts and/or isolated single components including various $\beta$-glucans and proteases from A. fumigatus have been used in most of the studies published on immune responses following exposure to mold ${ }^{[28,45-47]}$. Studies elucidating effects of viable spores or hyphae versus inactivated spores from A. fumigatus have reported that the growth stage (hyphae) and swelling spores have stronger pro-inflammatory potential than inactivated spores [28, 33, 38, 39]. In the present study, we have systematically characterized spore and hyphae fragment preparations from four species common in indoor environments and compared their pro-inflammatory potentials in different experimental models. We treated the samples with Xray ${ }^{[28]}$, since this procedure inactivates the specific mold stage without making changes to the structural features and/or surface molecules of fungal samples important for the immune responses (Øya el al. unpublished). An important finding is that the generalized hypothesis/dogma that "hyphae are more potent and give stronger pro-inflammatory responses than spores" does not necessarily hold true for all mold species, since A. versicolor and $S$. 
chartarum spores were found to be at least as potent as their hyphae counterpart. Furthermore, no single characterized component in the fungal preparations could predict the proinflammatory response to the particles.

We aimed to use fungal preparations as close as possible to naturally occurring airborne particles, thus spores were preferentially collected by air flow (aerosolized). Aerosolized spores were only obtained in sufficient quantity from A. fumigatus and P. chrysogenum. These preparations were slightly contaminated with hyphae fragments, 10 and 30\%, respectively as measured by number of fragments. Washed spores appeared to be smaller and/or have less dry weight than aerosolized spores, probably due to the presence of immature spores. The quality of the samples was considered very high except the spore samples from S. chartarum and partly P. chrysogenum. However, due to the relative large size of spores compared to the small size of the hyphal fragments, the purity of these samples was also estimated by volume which are closer related to mass. A relative high purity by mass was supported by the differences found in $\beta$-glucans, protease activities as well as their pro-inflammatory responses. It should also be noted that there are no direct correlations between inflammatory responses and particle number between the various fungal particle samples.

Several studies have reported that mycotoxins may suppress the inflammatory responses through cytotoxicity ${ }^{[48,49]}$. Thus, in order to remove any possible toxic effect of mycotoxins that might mask the pro-inflammatory potential, all spore preparations were washed after harvesting. Low levels of mycotoxins in the preparations after washing were confirmed by chemical analysis, and none of the preparations induced cytotoxicity. Small amounts of sterigmatocystin and roquefortine $\mathrm{C}$ were detected in some of the samples. Since neither of 
these have been reported to have pro-inflammatory effects, their presence did not explain the observed inflammatory responses.

Beta-glucans are mainly exposed during hyphae growth ${ }^{[50]}$. These carbohydrates are masked by rodlets, hydrophobins or $\alpha$-glucans on dormant spores ${ }^{[15,37,51]}$. In accordance with this, no or only low levels of $\beta$-glucans were found in preparations from A. fumigatus and $P$. chrysogenum, or A. versicolor and S. chartarum, respectively, whereas the levels in hyphae preparations were at least 10x higher.

Beta-glucans seem to elicit a strong immune response ${ }^{[50]}$, and some studies have linked exposure to $\beta$-glucans to inflammation-related health factors ${ }^{[52,53]}$. In line with this, we find a lower pro-inflammatory potential of $A$. fumigatus and $P$. chrysogenum spores when compared to their respective hyphae preparations. However, in the current study there was no correlation between the total level of $\beta$-glucans in the various hyphae preparations and their proinflammatory potential. Although the importance of that $\beta$-glucans for the triggering of immune responses is well documented, it is not likely that their total content necessarily reflects the proinflammatory potential. In addition to $\beta$-glucans, also other components of the cell wall contribute, including ergosterol and chitin. Most importantly, the availability of such molecules at the surface, as well as the topical structures of the particle are important determinants of the pro-inflammatory effects ${ }^{[50,54]}$.

Exposures to proteases, including those from molds, have been shown to stimulate inflammatory signaling pathways in airway cell models ${ }^{[45,55]}$. Often the triggering pathways involve crosstalk between PARs and various TLRs-mediated signaling pathways ${ }^{[56]}$. The characterization of the samples with regard to gelatin degrading protease activities revealed that 
the hyphae from A. fumigatus, P. chrysogenum and A. versicolor contained more protease activity than their respective washed/non-aerosolized spore preparations, corresponding with higher inflammatory potential. However, as the two $S$. chartarum samples contained high protease activity despite low pro-inflammatory potential, protease activity as such is not a good parameter for these inflammatory responses. A further characterization of the protease activity revealed that hyphae fragments of $A$. fumigatus contained a gelatin degrading serine protease, whereas hyphae fragments of $P$. chrysogenum consisted of a gelatin degrading metalloprotease. Thus, this serine protease could be more potent than the metalloprotease with respect to proinflammatory potential.

IL-1 $\beta$ and TNF- $\alpha$ are the early mediators of inflammatory reaction, which initiate and amplify a wide variety of effects associated with innate immunity and host responses to fungi ${ }^{[57,58]}$. Previous studies have suggested that phagocytes and epithelial cells are the primary target cells producing these inflammatory mediators in the lung ${ }^{[59]}$, while monocytes are recruited to the lung during inflammation ${ }^{[25]}$. The enhanced production of pro-inflammatory mediators is seen at non-cytotoxic concentrations, illustrating that inflammation/pro-inflammatory responses are the primary response to these fungal particles and that IL-1 $\beta$ may be secreted without membrane damage. The THP-1 macrophages model was the most sensitive model with respect to triggering inflammatory responses, followed by the undifferentiated THP-1 monocytes. This is in line with the notion that macrophages represent an important role of in the first line of defense towards molds ${ }^{[16]}$

Although the BEAS-2B cells did not increase the release of IL-1 $\beta$ and TNF- $\alpha$, it should be noted that at high concentrations hyphae fragments from all of the species increased the secretion of in particular IL-6 and for A. fumigatus and P. chrysogenum also IL-8. Furthermore, 
while the pro-inflammatory response in BEAS-2B appeared to be low, it is important to remember that the epithelial layer and its' mucus layer represent an important barrier towards various microbes. The differences in sensitivity between various models are greatly reflected by the fact that the more differentiated THP-1 macrophage stage has receptors involved in phagocytosis and/or fungi recognition that will trigger pro-inflammatory responses when compared to monocytes and epithelial cells. In accordance with this, the CLRs dectin-1 and 2 are highly expressed in macrophages ${ }^{[33,60]}$.

It is interesting to note that washed spores from A. fumigatus seem to give more proinflammatory effects, than the aerosolized spores. A possible explanation could be that more immature, smaller and/or lighter spores were collected by the washing technique than via the aerosolization. Mature spores will have a more complete hydrophobin layer and thicker cell wall making them less immune-reactive ${ }^{[15,51]}$.

With regard to A. fumigatus and $P$. chrysogenum, the hyphae preparations seem to have a larger potential to trigger IL-1 $\beta$ than their corresponding spore preparations, which is in accordance with the current dogma that spores are less pro-inflammatory than hyphae ${ }^{[6,15,61]}$. However, $A$. versicolor and $S$. chartarum washed spores seemed to give as least as large responses as their respective hyphae preparation. Dectin-1 recognizes $\beta$-glucans, whereas Dectin-2 binds to $\alpha$ mannans both being common in the fungal cell wall ${ }^{[33,60]}$. As the above mentioned receptors preferentially should bind to hyphae/germinating spores and not to resting spores, other receptors must also be involved/responsible for the spore-induced pro-inflammatory effects. Possibly THP-1 macrophages have receptors specifically recognizing some topical structures/epitopes on the spores irrespectively of their protease activity, mycotoxin or $\beta$-glucan content ${ }^{[33]}$. 
MMPs are secreted as pro-enzymes and their activity is carefully regulated. The longer exposure of both THP-1 monocytes and PMA-differentiated THP-1 macrophages to hyphae fragments resulted in increased release of proMMP-9. The basal release from the macrophages was found to be particularly high as also reported by others ${ }^{[21,22]}$. Although mold proteases may activate PAR-2, and PAR-2 is known to mediate upregulation of MMP-9 ${ }^{[62,63]}$, these two events are probably not linked, as the release was first seen after longer periods of exposure. More probably, the release of proMMP-9 is a secondary event due to release of IL-1 $\beta$ and/or TNF- $\alpha$ as these cytokines are known to trigger the release of MMPs ${ }^{[22]}$.

Based on equivalent dry weight concentrations of hyphae fragments added to cell cultures, the overall potential to stimulate the production of pro-inflammatory mediators decreased in the order A. fumigatus $>$ P. chrysogenum $>$ A. versicolor $>$ S. chartarum. By looking at their relative characteristic as determined by relative number, size and shape of particles, content of mycotoxins, $\beta$-glucan and crude proteases activity we find no single explanation to this observation. Thus, the pro-inflammatory reactions seems to be a complex combinations of several factors.

In the present study, pro-inflammatory effects of molds were detected at very low doses/concentrations (in the order of $10 \mathrm{ng} / \mathrm{cm}^{2}$ ) when compared to other studies ${ }^{[31,39,64]}$. This is rather relevant levels, as airborne concentrations in indoor environments are found to be in the range $10^{2}-10^{4}$ spores $/ \mathrm{m}^{3}{ }^{[6]}$. As chronic inflammatory responses as such are linked to adverse health effects, the present findings support the notion that mold exposure could be linked to health effects. However, further studies should be conducted in order to explore the relevance of these models and the possible unwanted implications of these type of responses. 
In conclusion, the present study has characterized aerosolized spores and hyphae fragments and washed/non-aerosolized spores from different fungal species common in moist indoor environments and compared their characteristics in relation to their inflammatory potential. The pro-inflammatory effects of molds were detected at low doses/concentrations supporting the notion that mold exposure could be linked to health effects. A. fumigatus seemed to be the most potent species. THP-1 macrophages were clearly the most sensitive model followed by THP-1 monocytes and BEAS-2B cells. There was no clear link between various sample characteristics and the observed pro-inflammatory response, illustrating the complexity of the many combined factors involved in such immune responses. Most interestingly, while the hyphae preparations of A. fumigatus and $P$. chrysogenum were more potent than the respective spore preparations, the opposite seems to be true for A. versicolor and S. chartarum.

\section{Acknowledgement}

The present work is a part of the project "Fungal particles in indoor air" that is funded by the Research Council of Norway: Grant number NFR196130/H10.

\section{References}

1. WHO, WHO Guidelines for Indoor Air Quality: Dampness and Mould. 2009, Geneva: World Health Organization.

2. Medicine, I.o., Human Health Effects, Damp Indoor Spaces and Health. National Academies Press, Washington, DC, 2004: p. 189-243.

3. Green, B.J., E.R. Tovey, J.K. Sercombe, F.M. Blachere, D.H. Beezhold, and D. Schmechel, Airborne fungal fragments and allergenicity. Med Mycol, 2006. 44 Suppl 1: p. S245-55.

4. Cramer, R.A., A. Rivera, and T.M. Hohl, Immune responses against Aspergillus fumigatus: what have we learned? Curr Opin Infect Dis, 2011. 24(4): p. 315-22.

5. Mendell, M.J., A.G. Mirer, K. Cheung, M. Tong, and J. Douwes, Respiratory and allergic health effects of dampness, mold, and dampness-related agents: a review of the epidemiologic evidence. Environ Health Perspect, 2011. 119(6): p. 748-56.

6. Eduard, W., Fungal spores: a critical review of the toxicological and epidemiological evidence as a basis for occupational exposure limit setting. Crit Rev Toxicol, 2009. 39(10): p. 799-864. 
7. Gorny, R.L., T. Reponen, K. Willeke, D. Schmechel, E. Robine, M. Boissier, and S.A. Grinshpun, Fungal fragments as indoor air biocontaminants. Appl Environ Microbiol, 2002. 68(7): p. 3522-31.

8. Afanou, K.A., A. Straumfors, A. Skogstad, T. Nilsen, O. Synnes, I. Skaar, L. Hjeljord, A. Tronsmo, B.J. Green, and W. Eduard, Submicronic Fungal Bioaerosols: High-Resolution Microscopic Characterization and Quantification. Applied and Environmental Microbiology, 2014. 80(22): p. 7122-7130.

9. Afanou, K.A., A. Straumfors, A. Skogstad, I. Skaar, L. Hjeljord, O. Skare, B.J. Green, A. Tronsmo, and W. Eduard, Profile and Morphology of Fungal Aerosols Characterized by Field Emission Scanning Electron Microscopy (FESEM). Aerosol Science and Technology, 2015. 49(6): p. 423-435.

10. Afanou, K.A., A. Straumfors, A. Skogstad, A.P. Nayak, I. Skaar, L. Hjeljord, A. Tronsmo, W. Eduard, and B.J. Green, Indirect Immunodetection of Fungal Fragments by Field Emission Scanning Electron Microscopy. Applied and Environmental Microbiology, 2015. 81(17): p. 5794-5803.

11. Lau, A.P.S., A.K.Y. Lee, C.K. Chan, and M. Fang, Ergosterol as a biomarker for the quantification of the fungal biomass in atmospheric aerosols. Atmospheric Environment, 2006. 40(2): p. 249-259.

12. Seo, S.C., T. Reponen, L. Levin, and S.A. Grinshpun, Size-fractionated (1-->3)-beta-D-glucan concentrations aerosolized from different moldy building materials. Sci Total Environ, 2009. 407(2): p. 806-14.

13. Pestka, J.J., Mechanisms of deoxynivalenol-induced gene expression and apoptosis. Food Addit Contam Part A Chem Anal Control Expo Risk Assess, 2008. 25(9): p. 1128-40.

14. Gammelsrud, A., A. Solhaug, B. Dendele, W.J. Sandberg, L. Ivanova, A. Kocbach Bolling, D. Lagadic-Gossmann, M. Refsnes, R. Becher, G. Eriksen, and J.A. Holme, Enniatin B-induced cell death and inflammatory responses in RAW 267.4 murine macrophages. Toxicol Appl Pharmacol, 2012. 261(1): p. 74-87.

15. Aimanianda, V., J. Bayry, S. Bozza, O. Kniemeyer, K. Perruccio, S.R. Elluru, C. Clavaud, S. Paris, A.A. Brakhage, S.V. Kaveri, L. Romani, and J.P. Latge, Surface hydrophobin prevents immune recognition of airborne fungal spores. Nature, 2009. 460(7259): p. 1117-21.

16. Heinekamp, T., H. Schmidt, K. Lapp, V. Pahtz, I. Shopova, N. Koster-Eiserfunke, T. Kruger, O. Kniemeyer, and A.A. Brakhage, Interference of Aspergillus fumigatus with the immune response. Semin Immunopathol, 2015. 37(2): p. 141-52.

17. Young, S.-H. and V. Castranova, Toxicology of 1 $\rightarrow$ 3-Beta-Glucans: Glucans as a Marker for Fungal Exposure, ed. S.-H.Y.a.V. Castranova. 2005, CRC Press: Taylor \& Francis Group, LLC.

18. Lopez-Otin, C. and J.S. Bond, Proteases: multifunctional enzymes in life and disease. J Biol Chem, 2008. 283(45): p. 30433-7.

19. Cawston, T.E. and A.J. Wilson, Understanding the role of tissue degrading enzymes and their inhibitors in development and disease. Best Pract Res Clin Rheumatol, 2006. 20(5): p. 9831002.

20. Rawlings, N.D., A.J. Barrett, and R. Finn, Twenty years of the MEROPS database of proteolytic enzymes, their substrates and inhibitors. Nucleic Acids Res, 2016. 44(D1): p. D343-50.

21. Goetzl, E.J., M.J. Banda, and D. Leppert, Matrix metalloproteinases in immunity. J Immunol, 1996. 156(1): p. 1-4.

22. Quiding-Jarbrink, M., D.A. Smith, and G.J. Bancroft, Production of matrix metalloproteinases in response to mycobacterial infection. Infect Immun, 2001. 69(9): p. 5661-70.

23. Espinosa, V. and A. Rivera, First Line of Defense: Innate Cell-Mediated Control of Pulmonary Aspergillosis. Front Microbiol, 2016. 7: p. 272.

24. Park, S.J. and B. Mehrad, Innate immunity to Aspergillus species. Clin Microbiol Rev, 2009. 22(4): p. 535-51.

25. Shi, C. and E.G. Pamer, Monocyte recruitment during infection and inflammation. Nat Rev Immunol, 2011. 11(11): p. 762-74. 
26. Portnoy, J.M., P.B. Williams, and C.S. Barnes, Innate Immune Responses to Fungal Allergens. Curr Allergy Asthma Rep, 2016. 16(9): p. 62.

27. Akira, S., S. Uematsu, and O. Takeuchi, Pathogen recognition and innate immunity. Cell, 2006. 124(4): p. 783-801.

28. Rodland, E.K., E. Ager-Wick, B. Halvorsen, F. Muller, and S.S. Froland, Toll like receptor 5 (TLR5) may be involved in the immunological response to Aspergillus fumigatus in vitro. Med Mycol, 2011. 49(4): p. 375-9.

29. Bhan, U., M.J. Newstead, X. Zeng, M.N. Ballinger, L.R. Standiford, and T.J. Standiford, Stachybotrys chartarum-induced hypersensitivity pneumonitis is TLR9 dependent. Am J Pathol, 2011. 179(6): p. 2779-87.

30. Reed, C.E. and H. Kita, The role of protease activation of inflammation in allergic respiratory diseases. J Allergy Clin Immunol, 2004. 114(5): p. 997-1008; quiz 1009.

31. Bozza, S., R. Gaziano, A. Spreca, A. Bacci, C. Montagnoli, P. di Francesco, and L. Romani, Dendritic cells transport conidia and hyphae of Aspergillus fumigatus from the airways to the draining lymph nodes and initiate disparate Th responses to the fungus. J Immunol, 2002. 168(3): p. 1362-71.

32. Bellocchio, S., C. Montagnoli, S. Bozza, R. Gaziano, G. Rossi, S.S. Mambula, A. Vecchi, A. Mantovani, S.M. Levitz, and L. Romani, The contribution of the Toll-like/IL-1 receptor superfamily to innate and adaptive immunity to fungal pathogens in vivo. J Immunol, 2004. 172(5): p. 3059-69.

33. Gersuk, G.M., D.M. Underhill, L. Zhu, and K.A. Marr, Dectin-1 and TLRs permit macrophages to distinguish between different Aspergillus fumigatus cellular states. J Immunol, 2006. 176(6): p. 3717-24.

34. Steele, C., R.R. Rapaka, A. Metz, S.M. Pop, D.L. Williams, S. Gordon, J.K. Kolls, and G.D. Brown, The beta-glucan receptor dectin-1 recognizes specific morphologies of Aspergillus fumigatus. PLoS Pathog, 2005. 1(4): p. e42.

35. Chai, L.Y., B.J. Kullberg, A.G. Vonk, A. Warris, A. Cambi, J.P. Latge, L.A. Joosten, J.W. van der Meer, and M.G. Netea, Modulation of Toll-like receptor 2 (TLR2) and TLR4 responses by Aspergillus fumigatus. Infect Immun, 2009. 77(5): p. 2184-92.

36. Feghali, C.A. and T.M. Wright, Cytokines in acute and chronic inflammation. Front Biosci, 1997. 2: p. d12-26.

37. Hohl, T.M., H.L. Van Epps, A. Rivera, L.A. Morgan, P.L. Chen, M. Feldmesser, and E.G. Pamer, Aspergillus fumigatus triggers inflammatory responses by stage-specific beta-glucan display. PLoS Pathog, 2005. 1(3): p. e30.

38. Bellanger, A.P., L. Millon, K. Khoufache, D. Rivollet, I. Bieche, I. Laurendeau, M. Vidaud, F. Botterel, and S. Bretagne, Aspergillus fumigatus germ tube growth and not conidia ingestion induces expression of inflammatory mediator genes in the human lung epithelial cell line A549. J Med Microbiol, 2009. 58(Pt 2): p. 174-9.

39. Rodland, E.K., T. Ueland, T.M. Pedersen, B. Halvorsen, F. Muller, P. Aukrust, and S.S. Froland, Activation of platelets by Aspergillus fumigatus and potential role of platelets in the immunopathogenesis of Aspergillosis. Infect Immun, 2010. 78(3): p. 1269-75.

40. Malla, N., E. Berg, A.D. Theocharis, G. Svineng, L. Uhlin-Hansen, and J.O. Winberg, In vitro reconstitution of complexes between pro-matrix metalloproteinase- 9 and the proteoglycans serglycin and versican. Febs j, 2013. 280(12): p. 2870-87.

41. Winberg, J.O., S.O. Kolset, E. Berg, and L. Uhlin-Hansen, Macrophages secrete matrix metalloproteinase 9 covalently linked to the core protein of chondroitin sulphate proteoglycans. J Mol Biol, 2000. 304(4): p. 669-80.

42. Malla, N., E. Berg, U. Moens, L. Uhlin-Hansen, and J.O. Winberg, Biosynthesis of promatrix metalloproteinase-9/chondroitin sulphate proteoglycan heteromer involves a Rottlerinsensitive pathway. PLoS One, 2011. 6(6): p. e20616. 
43. Malla, N., E. Berg, L. Uhlin-Hansen, and J.O. Winberg, Interaction of pro-matrix metalloproteinase-9/proteoglycan heteromer with gelatin and collagen. J Biol Chem, 2008. 283(20): p. 13652-65.

44. Zhou, J., P. Zhu, J.L. Jiang, Q. Zhang, Z.B. Wu, X.Y. Yao, H. Tang, N. Lu, Y. Yang, and Z.N. Chen, Involvement of CD147 in overexpression of MMP-2 and MMP-9 and enhancement of invasive potential of PMA-differentiated THP-1. BMC Cell Biol, 2005. 6(1): p. 25.

45. Kauffman, H.F., J.F. Tomee, M.A. van de Riet, A.J. Timmerman, and P. Borger, Proteasedependent activation of epithelial cells by fungal allergens leads to morphologic changes and cytokine production. J Allergy Clin Immunol, 2000. 105(6 Pt 1): p. 1185-93.

46. Kouzaki, H., S.M. O'Grady, C.B. Lawrence, and H. Kita, Proteases induce production of thymic stromal lymphopoietin by airway epithelial cells through protease-activated receptor-2. J Immunol, 2009. 183(2): p. 1427-34.

47. Pei, R. and C.K. Gunsch, Inflammatory cytokine gene expression in THP-1 cells exposed to Stachybotrys chartarum and Aspergillus versicolor. Environ Toxicol, 2013. 28(1): p. 51-60.

48. Despot, D.J., S. Kocsube, O. Bencsik, A. Kecskemeti, A. Szekeres, C. Vagvolgyi, J. Varga, and M.S. Klaric, Species diversity and cytotoxic potency of airborne sterigmatocystin-producing Aspergilli from the section Versicolores. Sci Total Environ, 2016. 562: p. 296-304.

49. Rosenblum Lichtenstein, J.H., Y.H. Hsu, I.M. Gavin, T.C. Donaghey, R.M. Molina, K.J. Thompson, C.L. Chi, B.S. Gillis, and J.D. Brain, Environmental mold and mycotoxin exposures elicit specific cytokine and chemokine responses. PLoS One, 2015. 10(5): p. e0126926.

50. Barreto-Bergter, E. and R.T. Figueiredo, Fungal glycans and the innate immune recognition. Front Cell Infect Microbiol, 2014. 4: p. 145.

51. Carrion Sde, J., S.M. Leal, Jr., M.A. Ghannoum, V. Aimanianda, J.P. Latge, and E. Pearlman, The RodA hydrophobin on Aspergillus fumigatus spores masks dectin-1- and dectin-2dependent responses and enhances fungal survival in vivo. J Immunol, 2013. 191(5): p. 2581 8.

52. Fogelmark, B., J. Thorn, and R. Rylander, Inhalation of (1-->3)-beta-D-glucan causes airway eosinophilia. Mediators Inflamm, 2001. 10(1): p. 13-9.

53. Rylander, R. and R.H. Lin, (1-->3)-beta-D-glucan - relationship to indoor air-related symptoms, allergy and asthma. Toxicology, 2000. 152(1-3): p. 47-52.

54. Mintz-Cole, R.A., E.B. Brandt, S.A. Bass, A.M. Gibson, T. Reponen, and G.K. Khurana Hershey, Surface availability of beta-glucans is critical determinant of host immune response to Cladosporium cladosporioides. J Allergy Clin Immunol, 2013. 132(1): p. 159-69.

55. Borger, P., G.H. Koeter, J.A. Timmerman, E. Vellenga, J.F. Tomee, and H.F. Kauffman, Proteases from Aspergillus fumigatus induce interleukin (IL)-6 and IL-8 production in airway epithelial cell lines by transcriptional mechanisms. J Infect Dis, 1999. 180(4): p. 1267-74.

56. Chaudhary, N. and K.A. Marr, Impact of Aspergillus fumigatus in allergic airway diseases. Clin Transl Allergy, 2011. 1(1): p. 4.

57. Roilides, E., A. Dimitriadou-Georgiadou, T. Sein, I. Kadiltsoglou, and T.J. Walsh, Tumor necrosis factor alpha enhances antifungal activities of polymorphonuclear and mononuclear phagocytes against Aspergillus fumigatus. Infect Immun, 1998. 66(12): p. 5999-6003.

58. Eder, C., Mechanisms of interleukin-1beta release. Immunobiology, 2009. 214(7): p. 543-53.

59. Huttunen, K., A. Hyvarinen, A. Nevalainen, H. Komulainen, and M.R. Hirvonen, Production of proinflammatory mediators by indoor air bacteria and fungal spores in mouse and human cell lines. Environ Health Perspect, 2003. 111(1): p. 85-92.

60. Margalit, A. and K. Kavanagh, The innate immune response to Aspergillus fumigatus at the alveolar surface. FEMS Microbiol Rev, 2015. 39(5): p. 670-87.

61. Gresnigt, M.S. and F.L. van de Veerdonk, The role of interleukin-1 family members in the host defence against Aspergillus fumigatus. Mycopathologia, 2014. 178(5-6): p. 395-401.

62. Vliagoftis, H., A. Schwingshackl, C.D. Milne, M. Duszyk, M.D. Hollenberg, J.L. Wallace, A.D. Befus, and R. Moqbel, Proteinase-activated receptor-2-mediated matrix metalloproteinase-9 release from airway epithelial cells. J Allergy Clin Immunol, 2000. 106(3): p. 537-45. 
63. Lee, S.E., J.M. Kim, S.K. Jeong, J.E. Jeon, H.J. Yoon, M.K. Jeong, and S.H. Lee, Proteaseactivated receptor-2 mediates the expression of inflammatory cytokines, antimicrobial peptides, and matrix metalloproteinases in keratinocytes in response to Propionibacterium acnes. Arch Dermatol Res, 2010. 302(10): p. 745-56.

64. Rakkestad, K.E., I. Skaar, V.E. Ansteinsson, A. Solhaug, J.A. Holme, J.J. Pestka, J.T. Samuelsen, H.J. Dahlman, J.K. Hongslo, and R. Becher, DNA damage and DNA damage responses in THP-1 monocytes after exposure to spores of either Stachybotrys chartarum or Aspergillus versicolor or to T-2 toxin. Toxicol Sci, 2010. 115(1): p. 140-55. 
Table 1: Distribution of fungal particle types in the various mold samples

\begin{tabular}{|c|c|c|c|c|c|c|c|c|c|c|}
\hline & \multicolumn{3}{|c|}{ Aspergillus fumigatus } & \multicolumn{3}{|c|}{ Penicillium chrysogenum } & \multicolumn{2}{|c|}{ Aspergillus versicolor } & \multicolumn{2}{|c|}{ Stachybotrys chartarum } \\
\hline & $\begin{array}{c}\text { Aerosolized } \\
\text { spores }\end{array}$ & $\begin{array}{l}\text { Washed } \\
\text { spores }\end{array}$ & $\begin{array}{l}\text { Hyphae } \\
\text { fragments }\end{array}$ & $\begin{array}{l}\text { Aerosolized } \\
\text { spores }\end{array}$ & $\begin{array}{l}\text { Washed } \\
\text { spores }\end{array}$ & $\begin{array}{l}\text { Hyphae } \\
\text { fragments }\end{array}$ & $\begin{array}{c}\text { Washed } \\
\text { spores }\end{array}$ & $\begin{array}{l}\text { Hyphae } \\
\text { fragments }\end{array}$ & $\begin{array}{l}\text { Washed } \\
\text { spores }\end{array}$ & $\begin{array}{l}\text { Hyphae } \\
\text { fragments }\end{array}$ \\
\hline Number counted & 200 & 201 & 200 & 201 & 204 & 201 & 201 & 204 & 214 & 201 \\
\hline \multicolumn{11}{|l|}{ Fragments, \% } \\
\hline $0.2-1 \mu \mathrm{m}$ & 4 & 0 & 5.5 & 1.5 & 0.5 & 9.5 & 0 & 4.4 & 10.8 & 9.5 \\
\hline $1-2 \mu \mathrm{m}$ & 3 & 0 & 28 & 18.9 & 2.9 & 16.9 & 1.5 & 5.4 & 29 & 27.4 \\
\hline $2-5 \mu \mathrm{m}$ & 3 & 1.5 & 36.5 & 9 & 3.9 & 43.3 & 1.5 & 28.4 & 28 & 27.4 \\
\hline $5-10 \mu \mathrm{m}$ & 0 & 1.5 & 19 & 1 & 0 & 17.4 & 1 & 30.4 & 5.1 & 12.9 \\
\hline$>10 \mu \mathrm{m}$ & 0 & 0 & 11 & 0 & 0 & 11.9 & 0 & 31.4 & 1.4 & 22.9 \\
\hline All & 10 & 3 & 100 & 30 & 4 & 99 & 4 & 100 & 74 & 100 \\
\hline \multicolumn{11}{|l|}{ Spores, \% } \\
\hline Single & 82 & 85.1 & 0 & 63.7 & 82.4 & 1 & 83.1 & 0 & 25.7 & 0 \\
\hline Aggregates of 2 & 6 & 10.5 & 0 & 4 & 6.4 & 0 & 10.5 & 0 & 0 & 0 \\
\hline Aggregates of 3 & 1.5 & 1 & 0 & 0.5 & 1.5 & 0 & 2 & 0 & 0 & 0 \\
\hline Aggregates of 4 & 0 & 0.5 & 0 & 0 & 2 & 0 & 0 & 0 & 0 & 0 \\
\hline Aggregates $\geq 5$ & 0.5 & 0 & 0 & 1.5 & 0.5 & 0 & 0.5 & 0 & 0 & 0 \\
\hline All & 90 & 97 & 0 & 70 & 96 & 1 & 96 & 0 & 26 & 0 \\
\hline Particles/mg & $0.31 \times 10^{8}$ & $0.30 \times 10^{8}$ & & $0.40 \times 10^{8}$ & $0.57 \times 10^{8}$ & & $0.44 \times 10^{8}$ & & $0.13 \times 10^{8}$ & \\
\hline Particles/mL & $9.52 \times 10^{5}$ & $2.90 \times 10^{6}$ & $1.23 \times 10^{6}$ & $7.57 \times 10^{5}$ & $1.47 \times 10^{6}$ & $1.85 \times 10^{6}$ & $2.83 \times 10^{6}$ & $1.72 \times 10^{6}$ & $2.26 \times 10^{6}$ & $1.34 \times 10^{6}$ \\
\hline
\end{tabular}


Table 2: Detected fungal metabolites in the particle fractions and estimated concentrations in methanol supplemented and sonicated suspensions (ng/mL)

\begin{tabular}{|c|c|c|c|c|c|c|c|c|c|c|}
\hline \multirow[b]{2}{*}{$\begin{array}{c}\text { Fungal } \\
\text { metabolite }\end{array}$} & \multicolumn{3}{|c|}{ Aspergillus fumigatus } & \multicolumn{3}{|c|}{ Penicillium chrysogenum } & \multicolumn{2}{|c|}{ Aspergillus versicolor } & \multicolumn{2}{|c|}{ Stachybotrys chartarum } \\
\hline & $\begin{array}{l}\text { Aerosolized } \\
\text { spores }\end{array}$ & $\begin{array}{l}\text { Washed } \\
\text { spores }\end{array}$ & $\begin{array}{l}\text { Hyphae } \\
\text { fragments }\end{array}$ & $\begin{array}{c}\text { Aerosolized } \\
\text { spores }\end{array}$ & $\begin{array}{l}\text { Washed } \\
\text { spores }\end{array}$ & $\begin{array}{l}\text { Hyphae } \\
\text { fragments }\end{array}$ & $\begin{array}{c}\text { Washed } \\
\text { spores }\end{array}$ & $\begin{array}{l}\text { Hyphae } \\
\text { fragments }\end{array}$ & $\begin{array}{l}\text { Washed } \\
\text { spores }\end{array}$ & $\begin{array}{l}\text { Hyphae } \\
\text { fragments }\end{array}$ \\
\hline Sterigmatocystin & - & - & - & - & - & - & 153 & + & - & - \\
\hline Methoxy- & & & & & & & & & & \\
\hline sterigmatocystin & - & - & - & - & - & - & $*$ & - & - & - \\
\hline Roquefortine $\mathrm{C}$ & - & - & - & 11.5 & 67.3 & 1.23 & - & - & - & - \\
\hline
\end{tabular}

(-): not detected; $(+)$ : detected, but not quantified; $(*)$ : tentatively identified, but no reference standard available 


\section{Figure legends}

Figure 1. Gelatinolytic proteases in spores and hyphae fragments from various molds. A) Representative gelatin zymography of the different mold samples. B) and C) Representative gelatin zymography of various mold samples where the gels were washed and incubated without (control), with $1 \mathrm{mM}$ Pefabloc, $10 \mathrm{mM}$ EDTA or $2.8 \mu \mathrm{M}$ E64, respectively. Arrows at the top of the gels indicate the bottom of the application well and the arrowheads the border between the stacking gel and the separating gel. The gelatin zymography shown in $\mathbf{A}$ ) is from the same gel. This is also the case for the control, Pefabloc, EDTA and E64 in B) and C).

Figure 2. Secretion of cytokines from THP-1 cells. A) THP-1 monocytes and B) PMAdifferentiated THP-1 cells were exposed to spores and hyphae fragments from different species for $24 \mathrm{~h}$ at concentrations indicated in the figures. IL-1 $\beta$ and TNF- $\alpha$ secretion are means \pm standard error of the mean (SEM) of separate experiment $n>3 ; * p<0.05$ control vs exposed. Statistical analysis was based on log-transformed data using analysis of variance with Dunnett's post hoc tests.

Figure 3. Secretion of gelatinolytic proteases from THP-1 cells. A) THP-1 monocytes were exposed to $10 \mu \mathrm{g}$ and B) PMA-differentiated THP-1 cells to $1 \mu \mathrm{g}$ dry weight $/ \mathrm{mL}$ of spores and hyphae fragments from various species for 72 h. A) Representative gelatin zymography of undiluted conditioned serum-free media from monocytes. B) Representative gelatin zymography of undiluted (upper panel) and five times diluted (lower panel) conditioned serumfree media from PMA-differentiated THP-1 cells. The various undiluted and diluted media was treated and loaded to the gel as described in Figure 1A). Standard (St) are identical with St2 in figure 1 and the molecular sizes in $\mathrm{kDa}$ are shown. Arrowheads show the border between the 
stacking gel and the separating gel. The gelatin zymography shown in B) lower panel are from the same gel. 
Fig. 1

A

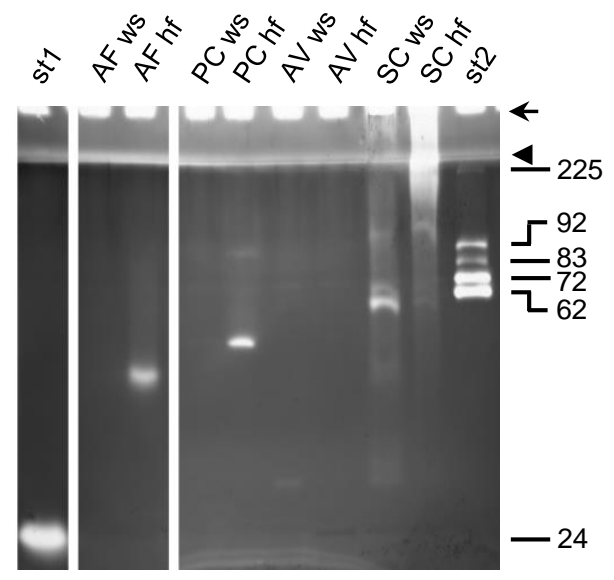

B

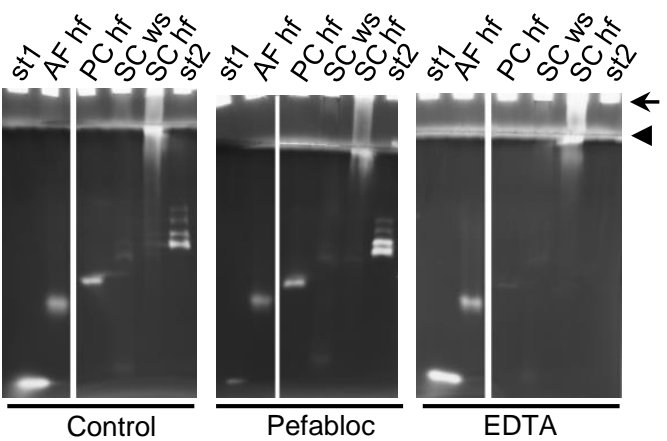

C

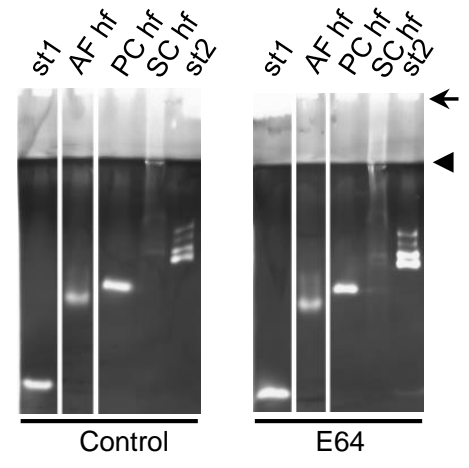


Fig. 2

A
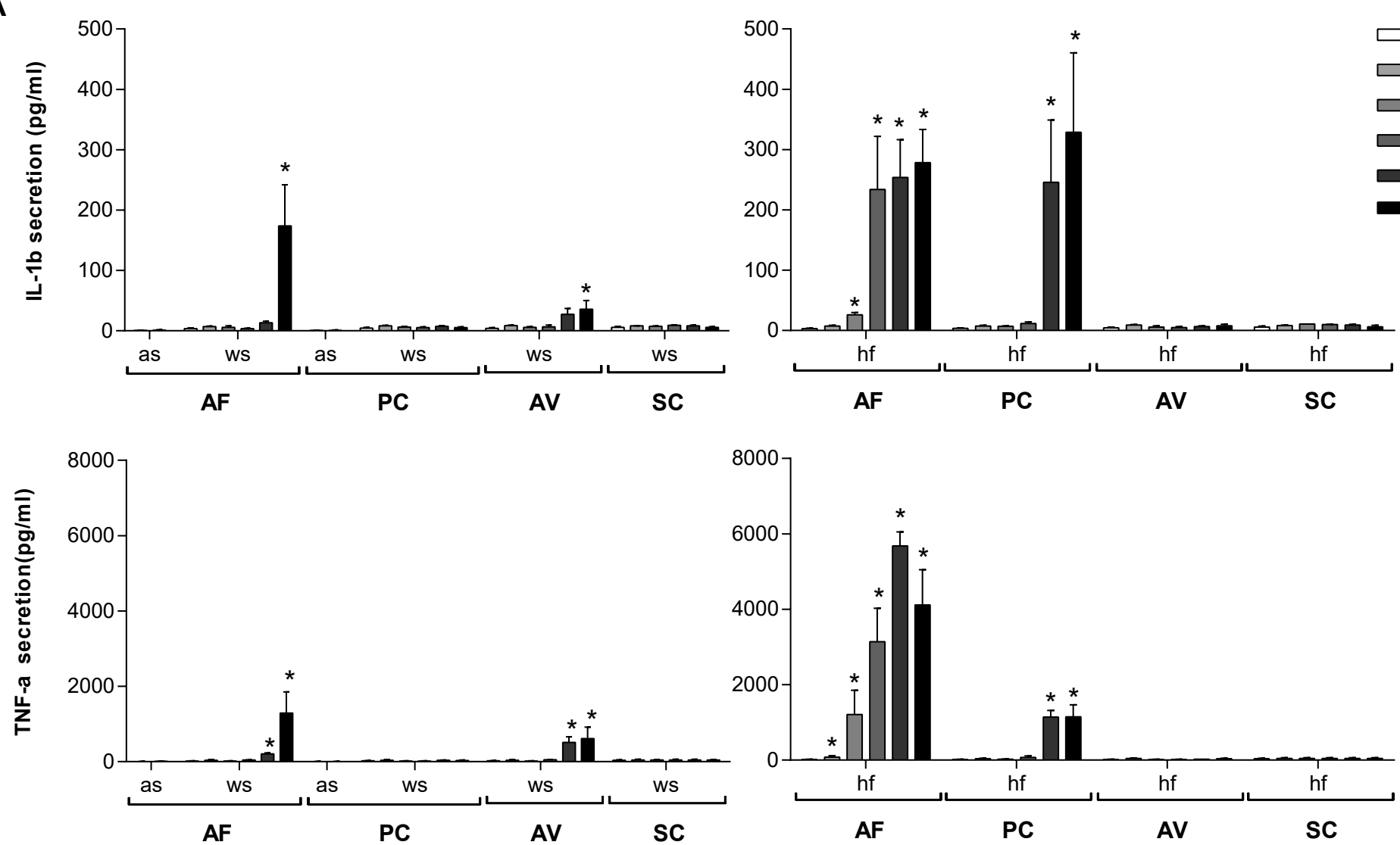

Aerosolized or washed spores (s)

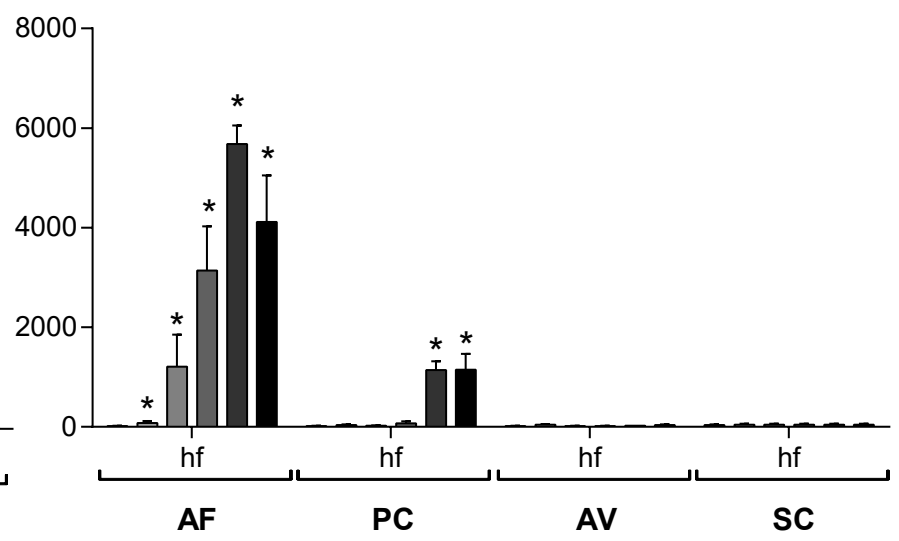

Hyphae fragments (hf)

B
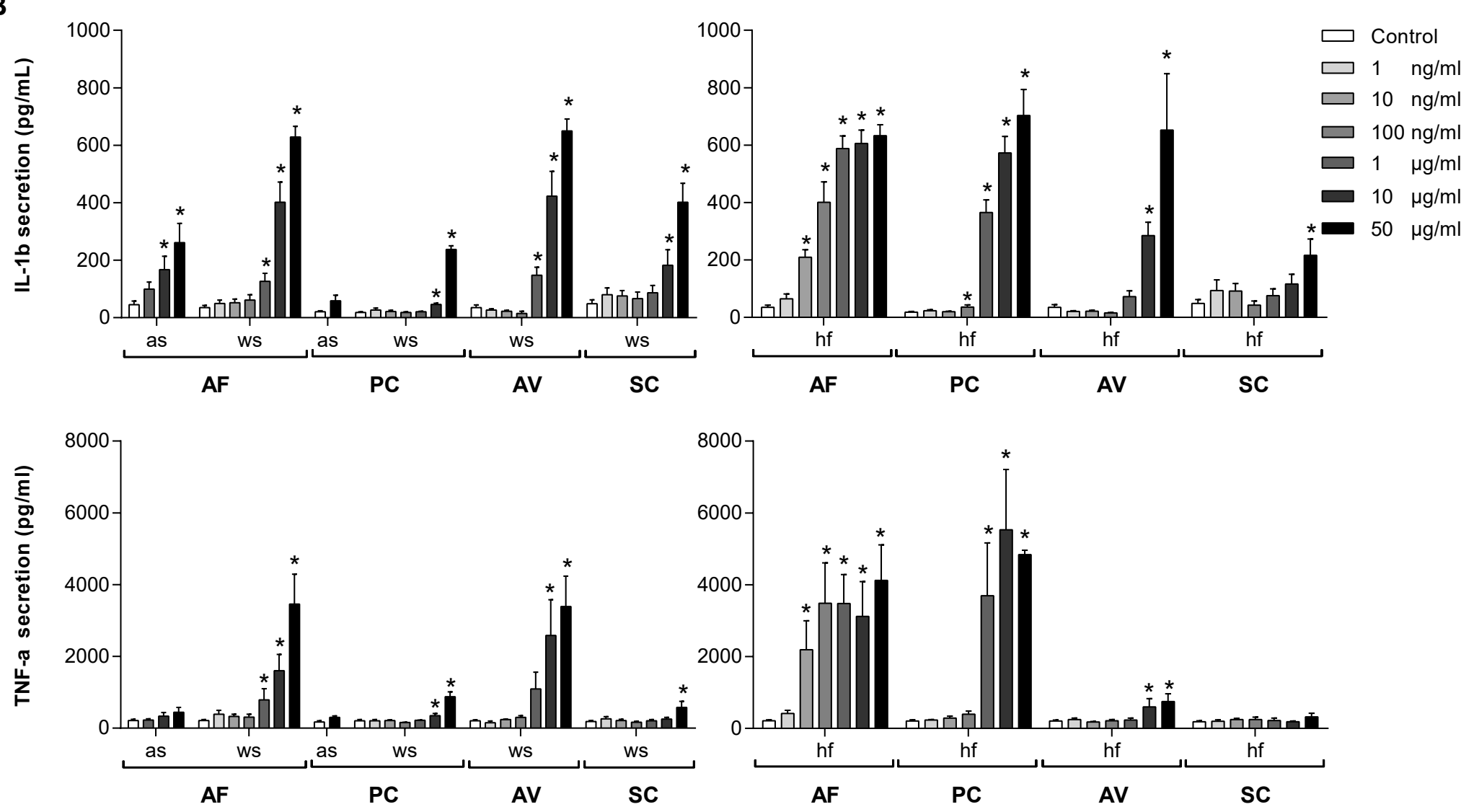

Aerosolized or washed spores (s)

Hyphae fragments (hf) 
Fig. 3
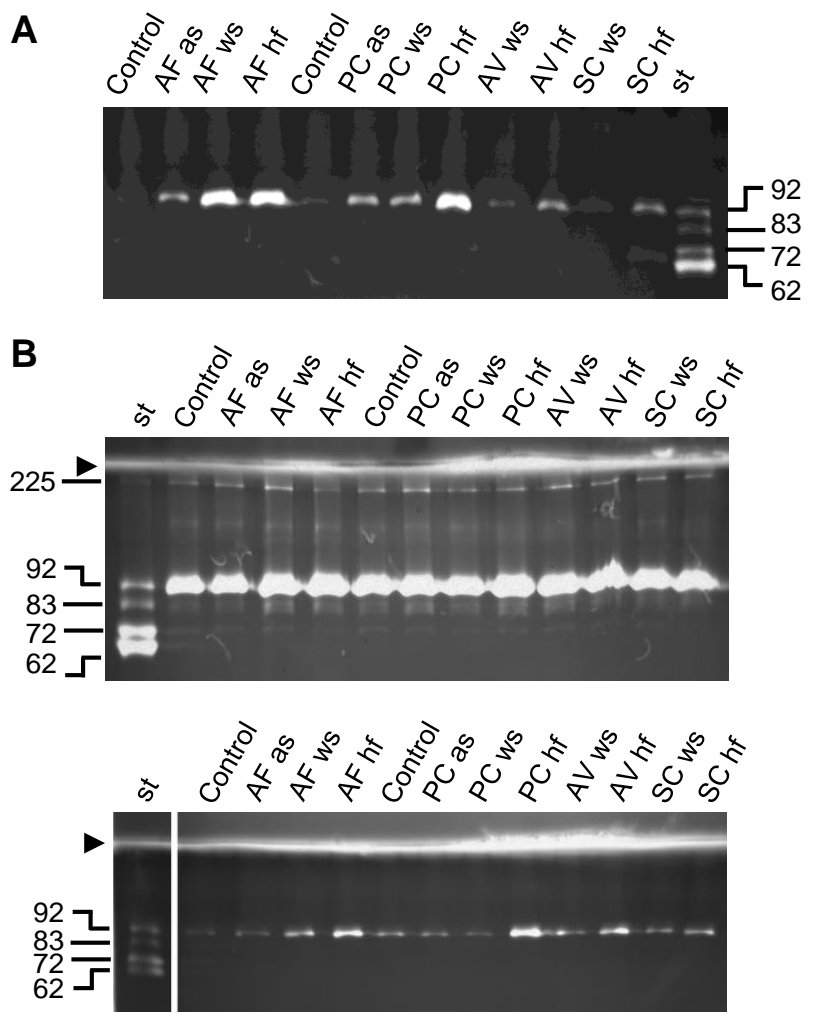


\section{Supplementary 1:}

\section{Materials and Methods}

\section{Chemicals}

Bacterial collagenase 1A (C9891; 760U/mg), rat tail collagen type I (C 8897), porcine skin gelatin (G 2500), 4-(2-aminoethyl) benzenesulfonyl fluoride (Pefabloc), E64 (E-3132), verrucarol, aflatoxin B, B1, G, G1, gliotoxin, roquefortine C, PR-toxin, bovine trypsin, pro matrix metalloproteinases (MMP)-2, were all from Sigma-Aldrich (St. Louis, MO, USA), while citrinin and sterigmatocystin were from Romer Labs (Tulln, Austria). Roridin A was a gift from James J. Pestka (Michigan State University). Phorbol 12-myristate 13-acetate (PMA) was from Calbiochem, EMD Chemicals, Inc (San Diego, CA, USA. ProMMP-9 was purified from PMA stimulated THP-1 cells as described previously ${ }^{[1]}$. LHC-9 cell culture medium was from Invitrogen (Carlsbad, CA, USA) and PureCol ${ }^{\mathrm{TM}}$ collagen from Advanced BioMatrix (Carlsbad, CA, USA). RPMI 1640 medium was from Lonza (Verviers, Belgium). Cytokine ELISA assays for TNF- $\alpha$ (Human TNF- $\alpha$ Cytoset), IL-6 (Human IL-6 Cytoset) and CXCL8 (Human IL-8 Cytoset) were purchased from Life Technologies (Camarillo, CA, USA). Cytokine ELISA assay for IL-1 $\beta$ (Human IL-1 $\beta$ Duoset) was purchased from R\&D Systems, Inc. (Minneapolis, MN, USA). All other chemicals used were purchased from commercial sources at the highest purity available.

\section{Particle preparation methods}

\section{Spores collected by liquid (washed spores; ws)}

Spores were gently scraped from respective fungal cultures grown on cellophane covered agar media by flooding with PBS containing $0.1 \%$ Tween 20 (PBST). The obtained spore suspension was resuspended by vortexing for $2 \times 30 \mathrm{~s}$ followed by sonication for $5 \mathrm{~min}$ in an ultrasonic bath at a frequency of $35 \mathrm{kHz}$ (Sonorex RK 510H, Bandalin Electric, Berlin, Germany). The spore 
suspension was then filtered through a $10 \mu \mathrm{m}$ nylon mesh filter mounted in Steriflip (Millipore, Tullagreen, Cork, Ireland) centrifuged at 5000×g for $10 \mathrm{~min}$ (Sigma 4k15, Osterode, Germany) and the pellet resuspended in appropriate volumes of LHC-9 cell culture medium. The prepared material was characterized by gravimetry and microscopy.

\section{Spores collected by air (aerosolized spores; as)}

Only the cultures from A. fumigatus and $P$. chrysogenum were subjected to aerosolization. Spores from the other isolates did not aerosolize sufficiently effective to give satisfactory amount of spores. The agar plates covered with fungal biomass were subjected to aerosolization at $20 \mathrm{~L} / \mathrm{min}$ in Stami Particle generator (SPG) following the procedure described by Afanou and co-workers ${ }^{[2]}$. The released spores were collected onto $0.4 \mu \mathrm{m}$ polycarbonate filters and eluted in a centrifuge tube by vortexing $(1 \times 5 \mathrm{~min})$ and sonicating $(1 \times 5 \mathrm{~min})$ in PBST. Suspended spores were filtered through $10 \mu \mathrm{m}$ nylon mesh and the filtrate was centrifuged as described in the section above. The supernatant was carefully discarded and the pellet resuspended in LHC-9 cell culture medium to achieve a final stock suspension of $30 \mathrm{mg} / \mathrm{mL}$. This suspension was further diluted and characterized by gravimetry and microscopy.

\section{Hyphae fragments $(h f)$}

Fungi were grown for 2- to 7-days on cellophane covered Sabouraud Dextrose Agar (SAB) plates based on our observations revealing late sporulation of the tested isolates. The samples were harvested prior to sporulation by scraping the hyphae mass into a polypropylene centrifuge tube. The biomass was freeze-dried using a Drywinner 1.0 to 6.0 (Heto, Denmark) connected to a RZ2 vacuum pump (Vacuubrand Gmbh, Wertheim, Germany) for 22 to $48 \mathrm{~h}$. The dried biomass was then ground in a RetchMM301 mixer mill (Retsch Gmbh, Haan, Germany), and the resulting hyphae powder was vacuumed through a cyclone (GK2.69 Cyclone; BGI, 
Waltham, MA, USA) operated in the respirable mode at $4.2 \mathrm{~L} / \mathrm{min}$ onto polycarbonate filters which were eluted as described in the section above. The obtained fragment pellet was resuspended in LHC-9 cell culture medium and incubated at $37^{\circ} \mathrm{C}$ for $20 \mathrm{~h}$.

\section{Supplementary Table S1: Different types of fungal samples and growth conditions}

\begin{tabular}{|c|c|c|c|c|c|c|c|c|c|c|c|}
\hline \multicolumn{3}{|c|}{ A. fumigatus } & \multicolumn{3}{|c|}{ A. versicolor } & \multicolumn{3}{|c|}{ P. chrysogenum } & \multicolumn{3}{|c|}{ S. chartarum } \\
\hline Types & Media & $\begin{array}{c}\text { Age } \\
\text { (days) }\end{array}$ & Types & Media & $\begin{array}{c}\text { Age } \\
\text { (days) }\end{array}$ & Types & Media & $\begin{array}{c}\text { Age } \\
\text { (days) }\end{array}$ & Types & Media & $\begin{array}{c}\text { Age } \\
\text { (days) }\end{array}$ \\
\hline $\begin{array}{c}\text { Aerosolized } \\
\text { spores }\end{array}$ & MEAC & 14 & - & - & - & $\begin{array}{c}\text { Aerosolized } \\
\text { spores }\end{array}$ & MEAC & 14 & - & - & - \\
\hline $\begin{array}{l}\text { Washed } \\
\text { spores }\end{array}$ & MEAC & 13 & $\begin{array}{l}\text { Washed } \\
\text { spores }\end{array}$ & MEAC & 21 & $\begin{array}{l}\text { Washed } \\
\text { spores }\end{array}$ & MEAC & 11 & $\begin{array}{l}\text { Washed } \\
\text { spores }\end{array}$ & PDAC & 10 \\
\hline $\begin{array}{l}\text { Freeze dried } \\
\text { hyphae } \\
\text { fragments }\end{array}$ & SABC & 4 & $\begin{array}{c}\text { Freeze dried } \\
\text { hyphae } \\
\text { fragments }\end{array}$ & SABC & 2 & $\begin{array}{c}\text { Freeze dried } \\
\text { hyphae } \\
\text { fragments }\end{array}$ & SABC & 7 & $\begin{array}{c}\text { Freeze } \\
\text { dried } \\
\text { hyphae } \\
\text { fragments }\end{array}$ & $\mathrm{SABC}$ & 3 \\
\hline
\end{tabular}

MEAC: cellophane covered malt extract agar; SABC: cellophane covered Sabouraud dextrose agar; PDAC: Cellophane covered potato dextrose agar. 


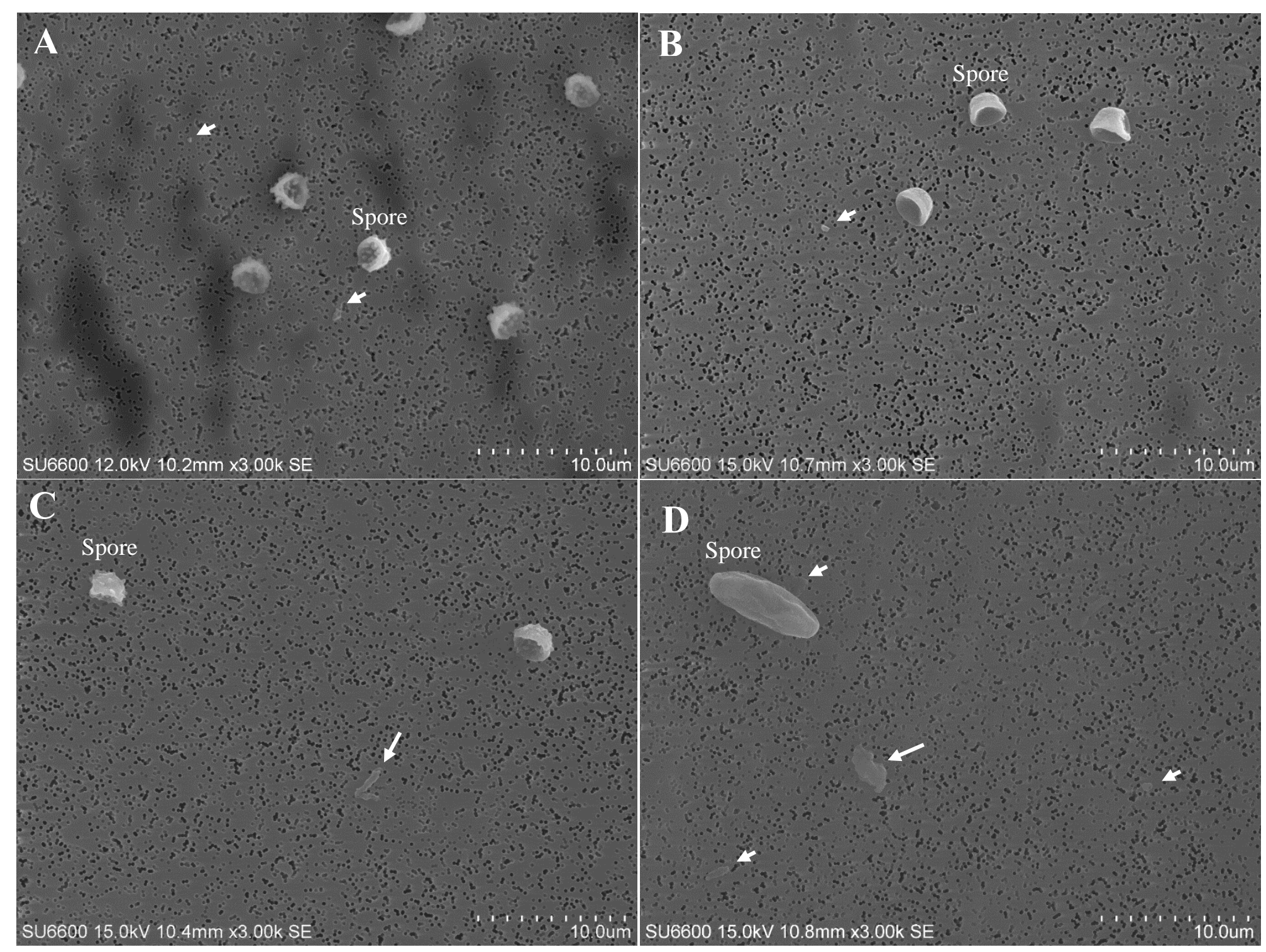

Supplementary Figure S1. Micrographs showing different types of fungal particles (spores and fragments (arrows)) in washed spore samples from A. fumigatus (A), P. chrysogenum (B); A. versicolor (C) and S. chartarum (D). 


\section{References}

1. Malla, N., E. Berg, L. Uhlin-Hansen, and J.O. Winberg, Interaction of pro-matrix metalloproteinase-9/proteoglycan heteromer with gelatin and collagen. J Biol Chem, 2008. 283(20): p. 13652-65.

2. Afanou, K.A., A. Straumfors, A. Skogstad, I. Skaar, L. Hjeljord, O. Skare, B.J. Green, A. Tronsmo, and W. Eduard, Profile and Morphology of Fungal Aerosols Characterized by Field Emission Scanning Electron Microscopy (FESEM). Aerosol Science and Technology, 2015. 49(6): p. 423-435. 


\section{Supplementary 2:}

\section{Mycotoxin methods}

Sample preparation for mycotoxin analyses

Aliquots (500 $\mu \mathrm{g}$ dry weight) of the suspensions containing washed spores, aerosolized spores or hyphae fragments in LHC-9 cell culture medium were transferred to Eppendorf tubes. Methanol (1:1) was added, the suspensions vortex shaken (15 s) and sonicated for $15 \mathrm{~min}$ at room temperature, and then, centrifuged at $15,000 \times \mathrm{g}$ for $5 \mathrm{~min}$ at room temperature. Supernatants were transferred to chromatography vials and sealed tight.

Base-hydrolysis of macrocyclic trichothecenes yielding verrucarol was tested using roridin A as a model compound and $0.1 \mathrm{M}$ carbonate buffer $(\mathrm{pH} 9.2), 2 \%$ ammonia or $0.1 \mathrm{M}$ sodium hydroxide as base. In the final protocol, $100 \mu \mathrm{L}$ of $0.2 \mathrm{M}$ sodium hydroxide was added to $100 \mu \mathrm{L}$-aliquots of the suspensions or $100 \mu \mathrm{L}$ of a 10:1 concentrated supernatant from washing of the spores (see below). The mixture was vortex shaken and heated to $80^{\circ} \mathrm{C}$ for $90 \mathrm{~min}$. The aliquots were centrifuged at $15,000 \times \mathrm{g}$ for $5 \mathrm{~min}$ and supernatants transferred to chromatography vials for LC-HRMS analysis.

The supernatant from washing of the spores was concentrated by solid-phase extraction on a $100 \mathrm{mg}$ Strata-X column (Phenomenex, Torrance, USA). The column was conditioned with $3 \mathrm{~mL}$ each of methanol and water, and then $10 \mathrm{~mL}$ of the supernatant was applied to the column. After washing with $3 \mathrm{~mL}$ of water and drying under vacuum, the column was eluted with $3 \mathrm{~mL}$ methanol. The solution was evaporated to dryness and the residue dissolved in $1 \mathrm{~mL}$ of water.

Liquid chromatography - high resolution mass spectrometry (LC-HRMS) procedure for mycotoxin analyses 
Separation of the suspensions containing aerosolized spores, washed spores, or hyphae fragments was achieved using a $100 \times 2.1 \mathrm{~mm}$, i.d. $2.6 \mu \mathrm{m}$ Kinetex EVO column (Phenomenex). Injection volumes were $1-5 \mu \mathrm{L}$. The mobile phase ( $400 \mu \mathrm{L} / \mathrm{min})$ consisted of $5 \mathrm{mM}$ ammonium acetate in $1 \%$ acetic acid (A), and $5 \mathrm{mM}$ ammonium acetate in 95:5 acetonitrile-water containing $1 \%$ acetic acid (B). The column was eluted isocratically with $5 \%$ B for 0.5 min followed by a linear gradient from 5-100\% B over $20 \mathrm{~min}$, followed by a 2 min hold and a return to 5\% B at $23 \mathrm{~min}$, and equilibration with 5\% B for 3 min using a Dionex UltiMate 3000 UPLC pump (Thermo Fisher Scientific, Waltham, MA). The detector was a Q-Exactive Fourier-transform high-resolution mass spectrometer (HRMS, Thermo Fisher Scientific) equipped with a heated electrospray ionization interface (HESI). The HRMS was run in positive and negative ion full-scan mode using fast polarity switching (i.e. alternating positive and negative ion scans), in the mass range $m / z, 250-800$. The mass resolution was set to 70,000 at $m / z 200$. The spray voltage was $4 \mathrm{kV}$, the transfer capillary temperature was $250^{\circ} \mathrm{C}$, and the sheath and auxiliary gas flow rates were 35 and 10 units, respectively. Exact values of $\mathrm{m} / \mathrm{z}$ used for extracted ion LC-HRMS chromatograms as well as mass errors were obtained using Xcalibur 2.2 or 3.0 (Thermo Fisher Scientific). The mass spectral characteristics of available reference toxins were studied by infusion $(5 \mu \mathrm{L} / \mathrm{min})$ of a $1-10 \mu \mathrm{g} / \mathrm{mL}$ solution into a mobile phase consisting of $1: 1 \mathrm{~A} / \mathrm{B}$.

The concentrations of sterigmatocystin and roquefortine $\mathrm{C}$ in the suspensions were estimated from external calibration of the LC-HRMS instrument using authentic standards dissolved in acetonitrile-water $(9: 1, \mathrm{v} / \mathrm{v})$. We did not make efforts to determine the limits of detection of target compounds. However, in our experience the quadrupole-Orbitrap hybrid instrument we used for detection of fungal metabolites in general provides limits of detection in the range $0.1-$ $5 \mathrm{ng} / \mathrm{mL}$ for small molecules. 
Available relevant reference standards for metabolites related to A. versicolor included sterigmatocystin and aflatoxins, which all afforded $[\mathrm{M}+\mathrm{H}]^{+}$ions using LC-HRMS in the positive ion mode. We also looked tentatively for methoxy-sterigmatocystin as well as versicolorins A and B by plotting extracted ion chromatograms for their $[\mathrm{M}+\mathrm{H}]^{+}$ions assuming that these analogues of aflatoxins and sterigmatocystin have the same MS ionization characteristics.

In order to test if methanol supplementation was necessary for the extraction of sterigmatocystin from the spores, or if the compound was also present in the aqueous medium we added water (1:1) to an aliquot of the spore suspension and placed it in an incubator at $37^{\circ} \mathrm{C}$ for $24 \mathrm{~h}$.

S. chartarum spore and hyphae suspensions were screened with regard to macrocyclic trichothecene mycotoxins (also known as type-D trichothecenes). Roridin A was used as a model compound and was found to afford $[\mathrm{M}+\text { acetate }]^{-}$ions upon electrospray ionization in the negative ion mode. The accurate $m / z$ of the $[\mathrm{M}-\mathrm{H}]^{-}$and $[\mathrm{M}+\text { acetate }]^{-}$ions were then calculated for a range of satratoxins $(\mathrm{F}, \mathrm{G}, \mathrm{H}$, isosatratoxin $\mathrm{F})$, roridins ( $\mathrm{A}$ and $\mathrm{E})$ and verrucarin $\mathrm{J}$ in order to plot extracted ion chromatograms from the full-scan LC-HRMS data. 


\section{Supplementary 3:}

\section{Mycotoxin results}

\section{Characterization of mycotoxin content}

The various samples containing aerosolized spores, washed spores and hyphae fragments from A. fumigatus were screened for the presence of gliotoxin. Gliotoxin was available as reference standard and afforded $[\mathrm{M}-\mathrm{H}]^{-}$ions using LC-HRMS in the negative ion mode. None of the samples contained gliotoxin in detectable concentrations. Furthermore, we plotted extracted ion chromatograms for sensible ions of fumitremorgins $\mathrm{A}-\mathrm{C}$, but could not detect any of these fungal metabolites.

Available metabolites related to P. chrysogenum included roquefortine C, PR-toxin and citrinin. Roquefortine afforded $[\mathrm{M}+\mathrm{H}]^{+}$and $[\mathrm{M}-\mathrm{H}]^{-}$ions upon positive and negative electrospray ionization, respectively. The former ions were, however, of approximately twice the intensity compared to the latter. Citrinin was found to afford primarily $\left[\mathrm{M}+\mathrm{H}_{2} \mathrm{O}-\mathrm{H}\right]^{-}$ions and PR-toxin $[\mathrm{M}+\mathrm{H}]^{+}$ions. The subsequent targeted analyses of the different suspensions of $P$. chrysogenum spores and hyphae fragments showed the presence of roquefortine $\mathrm{C}$ in all samples, but no citrinin or PR-toxin. The concentration of roquefortine $\mathrm{C}$ was highest in the medium suspension containing washed spores, and lowest in the medium suspension containing hyphae fragments (Table 2). We also looked for penicillin G by calculating the accurate $m / z$ of sensible ions and plotting extracted ion chromatograms but could not detect this compound.

Both the methanol supplemented medium suspension with washed spores and the suspension with hyphae fragments contained detectable amounts of sterigmatocystin (Table 2, Supplementary Figure S2). In the washed spore suspension, a nearby eluting compound identified as methoxy-sterigmatocystin based on its calculated elemental composition and LC- 
HRMS/MS data (Supplementary Figure S3). The relative peak area of the methoxy-analogue relative to sterigmatocystin was $1.5 \%$. The peak area of sterigmatocystin in the aqueous medium suspension was $7 \%$ of that of sterigmatocystin in the methanol supplemented medium, and hence we conclude that the concentration of the fungal toxin in the cell incubations was significantly lower than the estimated $153 \mathrm{ng} / \mathrm{mL}$ in the methanol supplemented spore suspension (Table 2).

None of the targeted trichothecenes were detected in the samples. In order to further investigate the presence of possible other type-D trichothecenes, we set up a protocol with the aim to basehydrolyze the ester linkages of the macrocyclic ring in any of the possibly present analogues. The expected product of the hydrolysis is verrucarol, which also was available as reference standard. The hydrolysis reaction was successfully tested using the roridin A (Supplementary Figure S4). However, base treatment of any of the S. chartarum samples, including the 10:1 concentrated supernatant from washing of the spores, did not result in the presence of verrucarol, and we thus conclude that none of the samples contained macrocyclic trichothecenes. 
Authentic standard of

sterigmatocystin

$(1 \mu \mathrm{g} / \mathrm{mL})$

suspension $(50 \%$

methanol, sonicated)

A. versicolor hyph
suspension $(50 \%$

methanol, sonicated)
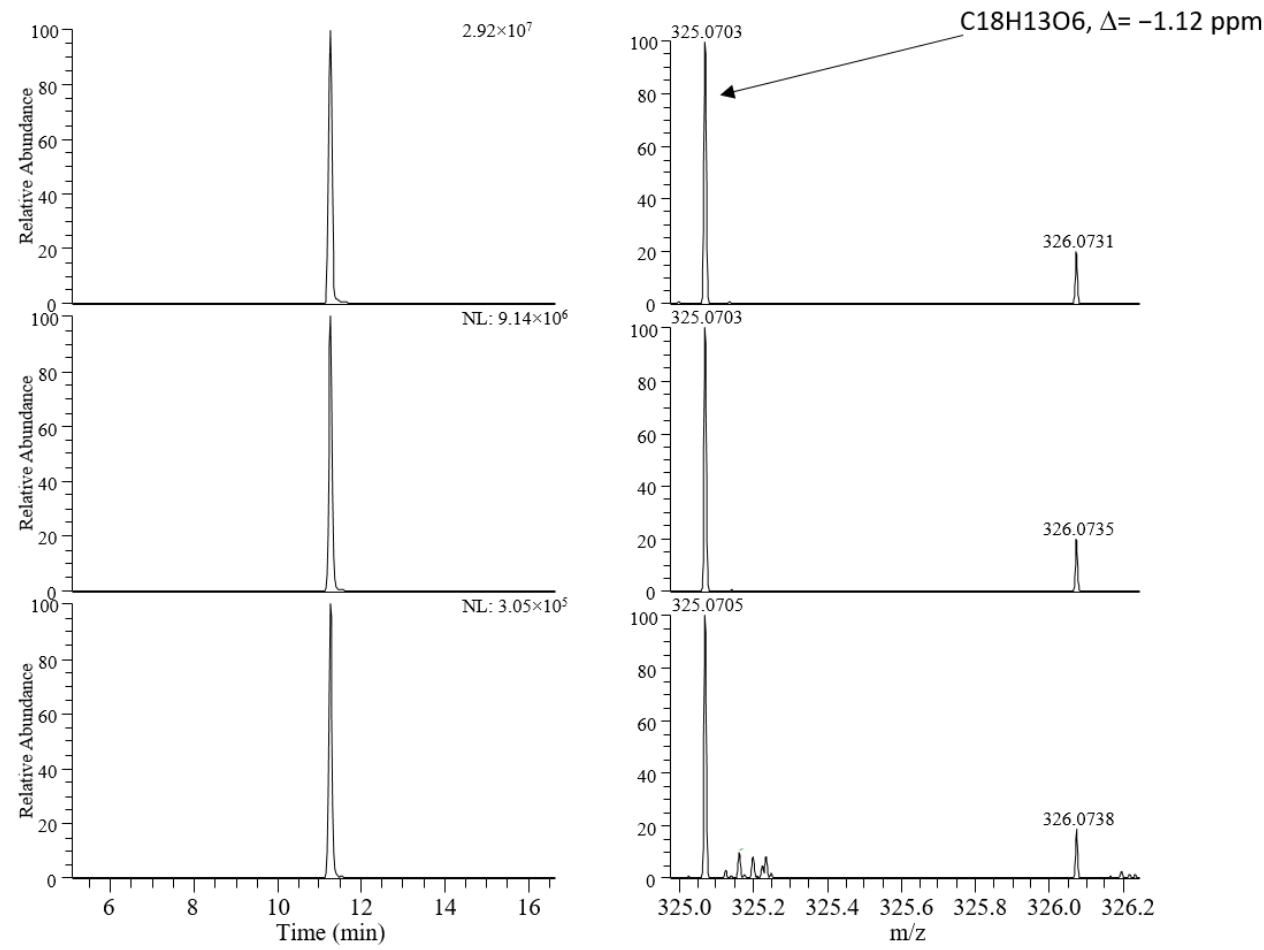

Supplementary Figure S2. LC-HRMS extracted ion chromatograms (left, $[\mathrm{M}+\mathrm{H}]^{+}, \pm 5 \mathrm{ppm}$ ) for sterigmatocystin in a standard solution as well as methanol supplemented and sonicated $A$. versicolor spore and hyphae samples, and mass spectrum of the protonated molecular ions. The number in the top right-hand corner of each chromatogram is the intensity of the highest peak in that chromatogram (arbitrary units). 

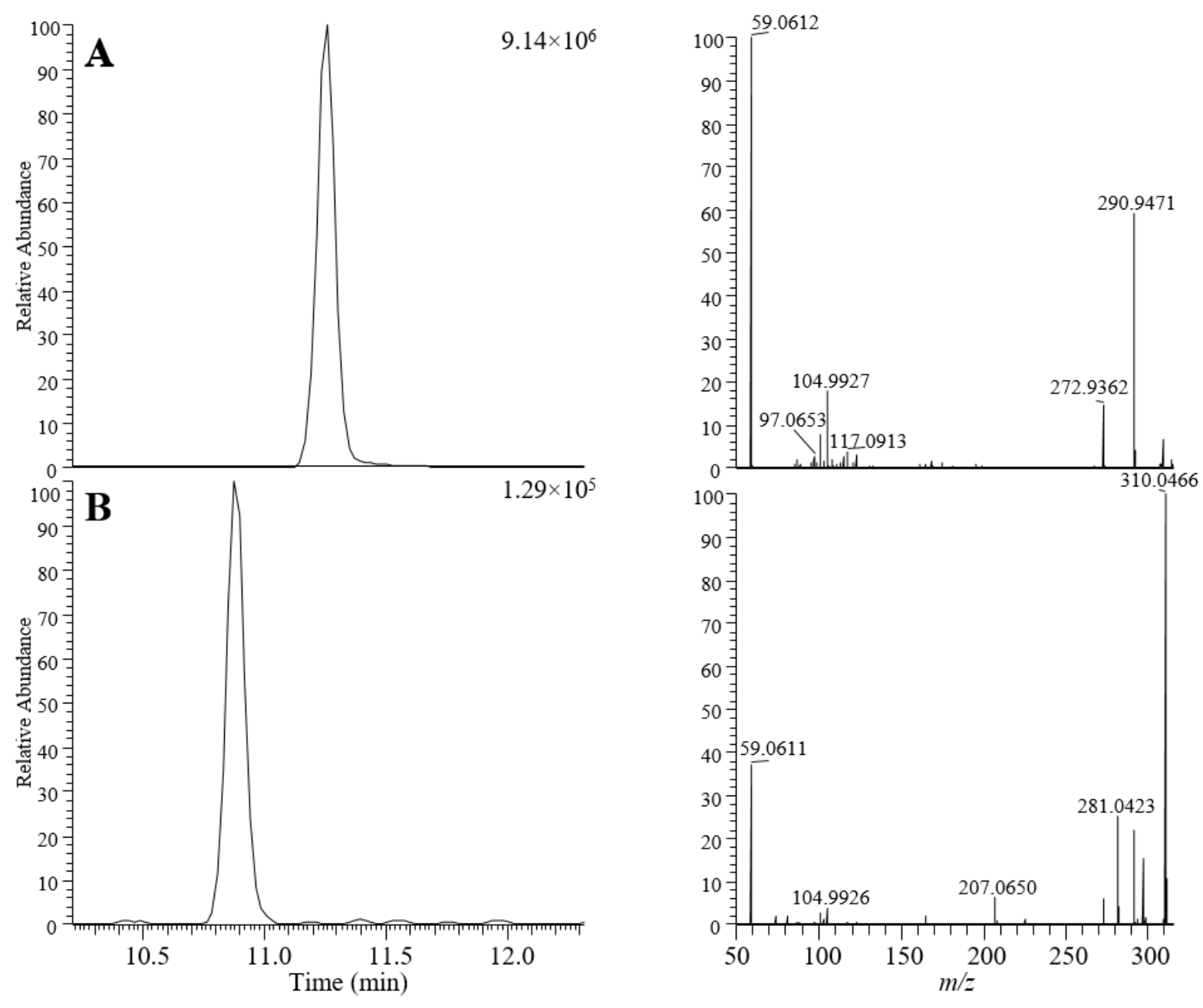

Supplementary Figure S3. LC-HRMS extracted ion chromatograms (left, $[\mathrm{M}+\mathrm{H}]^{+}, \pm 5 \mathrm{ppm}$ ) for sterigmatocystin (A) and putative methoxy-sterigmatocystin (B) in a methanol supplemented and sonicated A. versicolor spore samples, as well as product ion spectra from fragmentation of the protonated molecular ions. The number in the top right-hand corner of each chromatogram is the intensity of the highest peak in that chromatogram (arbitrary units). 
$0.1 \mathrm{M} \mathrm{NaOH}\left(80^{\circ} \mathrm{C}\right)$

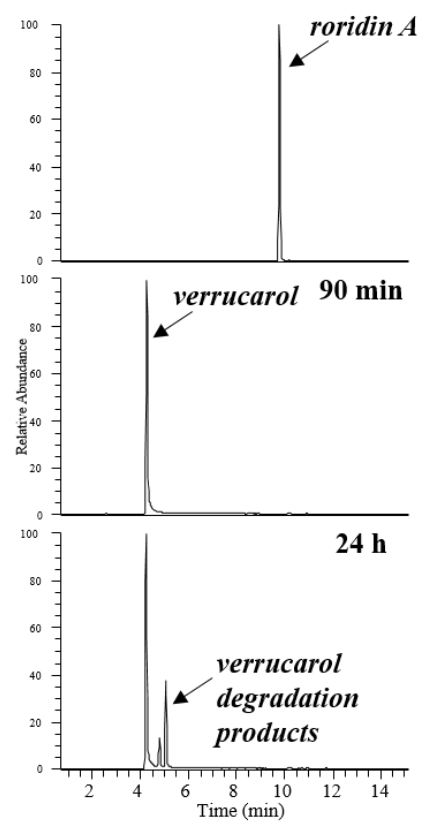

$2 \% \mathrm{NH}_{3}\left(90^{\circ} \mathrm{C}\right)$

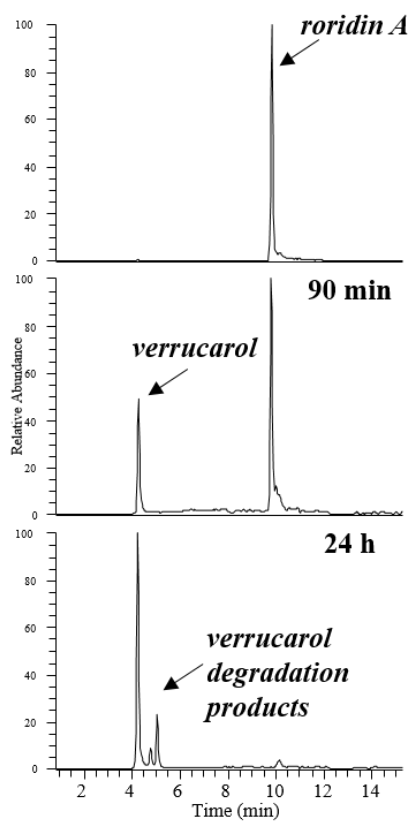

$0.2 \mathrm{M}$ carbonate buffer ( $\mathrm{pH} 9.4$, room temperature)

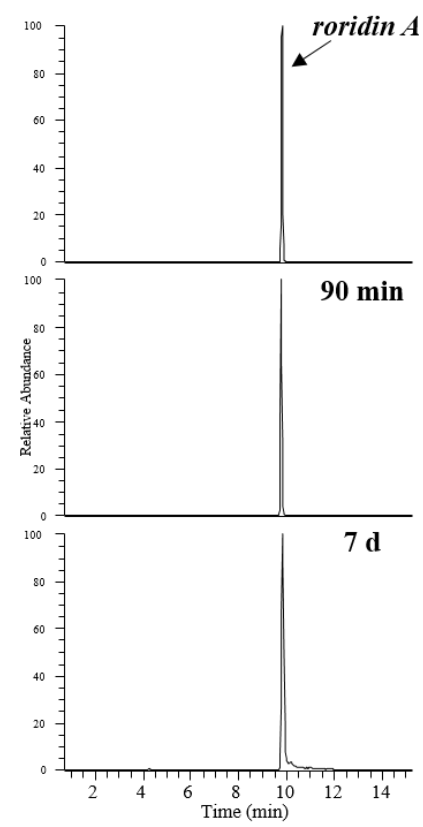

Supplementary Figure S4. LC-HRMS extracted ion chromatograms ([M+acetate $]^{-}, \pm 5$ ppm) from monitoring the base hydrolysis of roridin A in three different experiments employing different bases and temperatures. 


\section{Supplementary 4:}

Supplementary Table S2: $\beta$-1,3-glucans content in mold samples

\section{Mold samples}

A. fumigatus aerosolized spores

A. fumigatus hyphae fragments

P. chrysogenum aerosolized spores

P. chrysogenum hyphae fragments

A. versicolor washed spores

A. versicolor hyphae fragments

S. chartarum washed spores

S. chartarum hyphae fragments $\beta-1,3-$ glucans (ng/ml)

$<2.53$

244

$<2.53$

400

45.7

$>404$

45.5

$>404$ 

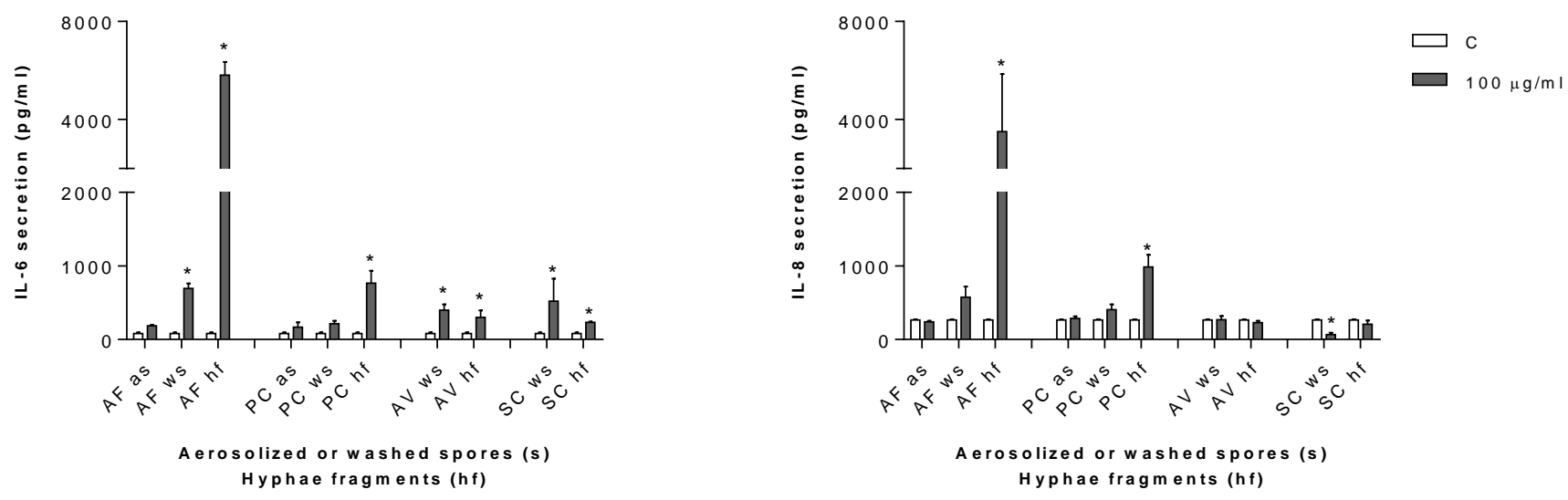

Supplementary Figure S5. Cytokine/chemokine secretion in BEAS-2B cells induced by different molds. Cells were exposed to 0 or $100 \mu \mathrm{g}$ dry weight $/ \mathrm{mL}$ of spores or hyphae fragments from various species for $24 \mathrm{~h}$. A) IL-6 secretion by ELISA after $24 \mathrm{~h}$ exposure of $100 \mu \mathrm{g}$ dry weight $/ \mathrm{mL}$ different species of mold in BEAS-2B cells. B) IL-8 secretion by ELISA after $24 \mathrm{~h}$ exposure of $100 \mu \mathrm{g} / \mathrm{mL}$ dry weight different species of mold in BEAS-2B cells. Protein release in cell culture supernatants was determined by ELISA. Bars represent protein levels detected in each separate experiment (mean \pm SEM, $n=3$ ). * $\mathrm{p}<0.05$ control vs exposed. Statistical analysis was based on log-transformed data using analysis of variance with Dunnett's post hoc tests. 